\title{
1 BBX proteins promote HY5-mediated UVR8 signaling in Arabidopsis
}

2

3 Roman Podolec ${ }^{1,2 * \dagger}$, Timothée B. Wagnon ${ }^{1 *}$, Manuela Leonardelli ${ }^{1}$, Henrik Johansson ${ }^{3}$, and 4 Roman Ulm ${ }^{1,2 \dagger}$

5

$6{ }^{1}$ Department of Botany and Plant Biology, Section of Biology, Faculty of Sciences, University of

7 Geneva, CH-1211 Geneva 4, Switzerland

8 Institute of Genetics and Genomics of Geneva (iGE3), University of Geneva, Geneva,

$9 \quad$ Switzerland

10 3nstitute of Biology/Applied Genetics, Dahlem Centre of Plant Sciences (DCPS), Freie

11 Universität Berlin, Berlin, Germany

12

13 ORCID IDs: 0000-0003-2998-7892 (R.P.), 0000-0002-9760-2408 (T.B.W.), 0000-0001-6215-

141478 (M.L.), 0000-0002-8965-9500 (H.J.), 0000-0001-8014-7392 (R.U.)

15

$16 *$ these authors contributed equally to this work (R.P., T.B.W.).

$17 \dagger$ †uthors for correspondence: roman.podolec@unige.ch (R.P.) and roman.ulm@unige.ch (R.U.)

18

19 Short title: UVR8 signaling involves BBX proteins 


\section{ABSTRACT}

21 Plants undergo photomorphogenic development in the presence of light. Photomorphogenesis is

22 repressed by the E3 ubiquitin ligase CONSTITUTIVELY PHOTOMORPHOGENIC1 (COP1),

23 which binds substrates through their valine-proline (VP) motifs. The UV RESISTANCE LOCUS8

24 (UVR8) photoreceptor senses UV-B and inhibits COP1 through cooperative binding of its own

25 VP motif mimicry and its photosensing core to COP1, thereby preventing COP1 binding to

26 substrates, including the bZIP transcriptional regulator ELONGATED HYPOCOTYL5 (HY5). As

27 a key promoter of visible light and UV-B photomorphogenesis, HY5 functions together with the

28 B-box family transcription factors BBX20-22 that were recently described as HY5 rate-limiting coactivators under red light. Here we describe a hypermorphic bbx21-3D mutant with enhanced photomorphogenesis, which carries a proline-314 to leucine mutation in the VP motif that impairs interaction with and regulation through COP1. We show that BBX21 and BBX22 are UVR8-

32 dependently stabilized after UV-B exposure, which is counteracted by a repressor induced by 33 HY5/BBX activity. bbx20 bbx21 bbx22 mutants under UV-B are impaired in hypocotyl growth 34 inhibition, photoprotective pigment accumulation, and expression of several HY5-dependent 35 genes. We conclude that BBX20-22 importantly contribute to HY5 activity in a subset of UV-B 36 responses, but that additional, presently unknown coactivators for HY5 are functional in early 37 UVR8 signaling. 


\section{INTRODUCTION}

Plants integrate light signals through a number of photoreceptor-initiated signaling pathways (Kami et al., 2010; Galvao and Fankhauser, 2015; Demarsy et al., 2018; Podolec et al., 2021a), which enables optimized growth in changing environments. When exposed to light, including ultraviolet-B (UV-B), seedlings undergo a developmental program termed photomorphogenesis, characterized by features such as hypocotyl growth inhibition, anthocyanin and flavonoid pigment accumulation, and cotyledon expansion (Kami et al., 2010; Galvao and Fankhauser, 2015). In Arabidopsis (Arabidopsis thaliana), UV-B radiation $(280-315 \mathrm{~nm})$ is sensed by the photoreceptor UV RESISTANCE LOCUS8 (UVR8) that induces a signaling pathway resulting in UV-B acclimation and thus enhanced UV-B tolerance (Kliebenstein et al., 2002; Favory et al., 2009; Rizzini et al., 2011; Podolec et al., 2021a; Rai et al., 2021). UVR8 is homodimeric in its inactive ground state, but monomerizes and becomes activated upon UV-B photon reception (Rizzini et al., 2011). A valine-proline (VP) motif in activated UVR8 represses the activity of the E3 ubiquitin ligase CONSTITUTIVELY PHOTOMORPHOGENIC1 (COP1) by competitively interacting with the binding site of COP1 substrates (Favory et al., 2009; Cloix et al., 2012; Yin et al., 2015; Lau et al., 2019).

Prominent among COP1 substrates is ELONGATED HYPOCOTYL5 (HY5), a key photomorphogenesis-promoting bZIP transcriptional regulator that induces expression of many UV-B-activated genes (Ulm et al., 2004; Brown et al., 2005; Oravecz et al., 2006). Upon UV-B exposure, HY5 is transcriptionally induced and the HY5 protein is stabilized (Ulm et al., 2004; Brown et al., 2005; Favory et al., 2009; Huang et al., 2013). However, the HY5 mode of action in UV-B signaling is not fully understood as it lacks a functional transcriptional activation domain (Ang et al., 1998; Stracke et al., 2010; Burko et al., 2020). Nevertheless, HY5 binds the promoters of many UV-B-induced genes and is required for their UV-B-induced expression (Ulm et al., 2004; Brown et al., 2005; Oravecz et al., 2006; Brown and Jenkins, 2008; Stracke et al., 2010; Binkert et al., 2014). Thereby, HY5 promotes UV-B responses such as hypocotyl growth inhibition, anthocyanin and flavonoid pigment accumulation, and ultimately stress acclimation (Brown et al., 2005; Oravecz et al., 2006; Favory et al., 2009; Huang et al., 2012). HY5-regulated genes include those encoding REPRESSOR OF UV-B PHOTOMORPHOGENESIS1 (RUP1) and RUP2, which are crucial negative feedback regulators facilitating UVR8 ground state reversion by redimerization (Gruber et al., 2010; Heijde and Ulm, 2013). In agreement, rup1 rup2 display strong 
UV-B hypersensitivity, further supported by the phenotype of the UVR8 $8^{\mathrm{G} 101 \mathrm{~S}}$ mutant variant that

70

71 is constitutively monomeric in vivo and underlies the enhanced UV-B photomorphogenic phenotype of the $u v r 8-17 D$ mutant allele (Podolec et al., 2021b).

Recently, the transactivation domain-containing, B-box zinc-finger transcription factors BBX20-22 were characterized as coregulators of HY5 under red light, interacting with HY5 and providing it with transcriptional activity (Bursch et al., 2020). BBX20-22, as well as BBX23, another member of class IV of the B-box family, have been described as positive regulators of photomorphogenesis (Datta et al., 2007; Chang et al., 2008; Datta et al., 2008; Chang et al., 2011; Fan et al., 2012; Wei et al., 2016; Zhang et al., 2017), whereas two other members of the subfamily, namely BBX24 and BBX25, play a repressive role (Indorf et al., 2007; Yan et al., 2011; Jiang et al., 2012; Gangappa et al., 2013; Crocco et al., 2015). In darkness, BBX20-25 are degraded via COP1, whereas they are stabilized in the light (Indorf et al., 2007; Chang et al., 2011; Fan et al., 2012; Jiang et al., 2012; Gangappa et al., 2013; Xu et al., 2016; Zhang et al., 2017; Job et al., 2018). BBX21, BBX24, and BBX25 contain experimentally defined COP1-interacting VP motifs, similar to other COP1 targets (Holm et al., 2001; Yan et al., 2011; Lau et al., 2019; Bursch et al., 2020). Moreover, HY5 has been suggested to repress BBX22 accumulation in an unknown manner (Chang et al., 2011). Whereas the negative regulator BBX24 has been implicated in UV-B signaling by suppressing HY5 activity (Jiang et al., 2012), it is not known whether the class IV BBX family positive regulators play a role in UV-B signaling in Arabidopsis. However, the involvement of functional homologs of class IV BBX positive regulators in UV-B signaling and anthocyanin biosynthesis has been reported in apple (Malus $\times$ domestica) in transgenic overexpression and suppression lines (Bai et al., 2014; Fang et al., 2019).

In this work, we identified and characterized a novel gain-of-function mutant of BBX21, namely $b b \times 21-3 D$, that contains a hypermorphic proline-314 to leucine (P314L) mutation in its VP motif. Phenotypic analyses suggested that the enhanced photomorphogenesis of this mutant is linked to increased activity of BBX21 $1^{\mathrm{P} 314 \mathrm{~L}}$ and impaired negative regulation by COP1. We further explored the role of the BBX20-22 subfamily in UV-B photomorphogenesis and found that BBX proteins are regulated at both transcriptional and post-translational levels under UV-B. We uncovered a HY5/BBX-mediated negative feedback mechanism affecting BBX protein stability. Moreover, a bbx20 bbx21 bbx22 mutant showed a lack of flavonoid and anthocyanin accumulation, impaired inhibition of hypocotyl elongation, and reduced marker gene expression under UV-B. 
bioRxiv preprint doi: https://doi.org/10.1101/2021.10.14.464399; this version posted October 14, 2021. The copyright holder for this preprint (which was not certified by peer review) is the author/funder, who has granted bioRxiv a license to display the preprint in perpetuity. It is made available under aCC-BY-NC-ND 4.0 International license.

100 Collectively, our work reveals that BBX20-22 play an important role in promoting HY5-mediated 101 UV-B responses. 
102

103

104

105

106

107

108

109

110

111

112

113

114

115

116

117

118

119

120

121

122

123

124

125

126

127

128

129

130

131

132

\section{RESULTS}

$b b x 21-3 D$ is a gain-of-function mutant of $B B X 21$ with an enhanced photomorphogenic response

In a hypocotyl length-based EMS mutant screen for altered UV-B photomorphogenesis (Podolec et al., 2021b), we discovered a mutant with an enhanced photomorphogenic response under white light with supplemental UV-B (mutant $b b \times 21-3 D$ in Fig. 1A,B). However, the manifestation of this phenotype under white light in the absence of supplemental UV-B indicated that it was not specific to UV-B (Fig. 1A,B). A causative transition mutation in $A T 1 G 75540$ (BBX21) was identified by whole-genome sequencing of a bulk segregant population; this mutation alters the VP motif of BBX21, namely proline-314 to leucine, resulting in a BBX21 $1^{\mathrm{P} 14 \mathrm{~L}}$ variant (Supplemental Fig. S1A). The novel mutant allele showed a dominant hypocotyl phenotype (Fig. 1C), and hence was named $b b \times 21-3 D$. To verify that the enhanced photomorphogenic phenotype of $b b x 21-3 D$ was caused by the BBX2 $1^{\mathrm{P} 314 \mathrm{~L}}$ mutation, we used CRISPR/Cas9 to knockout $B B X 21$ in the $b b \times 21-3 D$ background. Unlike $b b \times 21-3 D$, a $B B X 21$ knockout allele in $b b x 21-3 D$ showed an elongated hypocotyl phenotype comparable to the $b b \times 21-1$ null allele as well as the $b b \times 21^{K O}$ CRISPR/Cas9 knockout allele in the Col-0 wild-type background generated here (Supplemental Fig. S1B,C). These data support that BBX21 $1^{\mathrm{P} 314 \mathrm{~L}}$ is the causative mutation for the hypermorphic phenotype of $b b \times 21-3 D$. To further confirm that the $b b \times 21-3 D$ phenotype is due to the loss of a functional VP motif, we used CRISPR/Cas9 to mutate the C-terminus of BBX21 in a wild-type background (Supplemental Fig. S1B). Indeed, a premature stop codon causing a C-terminal deletion removing the BBX21 VP motif mimicked the short-hypocotyl phenotype of $b b \times 21-3 D$ (Supplemental Fig. S1D), supporting that the VP motif is crucial to negatively regulate BBX21 activity.

Whereas bbx21-3D showed an enhanced photomorphogenic response in both -UV-B and +UV-B conditions, the $b b \times 21-1$ null mutant exhibited slightly reduced hypocotyl growth inhibition in the presence of UV-B (Fig. 1A,B). Consistently, anthocyanin accumulation was enhanced in bbx21-3D in both $-\mathrm{UV}-\mathrm{B}$ and $+\mathrm{UV}-\mathrm{B}$ conditions compared to wild type, but was reduced relative to wild type under +UV-B in bbx21-1 (Fig. 1D). UV-B-induced accumulation of CHALCONE SYNTHASE (CHS), a key enzyme for phenylpropanoid biosynthesis, was also enhanced in $b b \times 21$ $3 D$ relative to wild type, whereas it accumulated to lower levels in the bbx21-1 null mutant (Fig. 1E). In $b b \times 21-3 D, U V-B-i n d u c e d ~ H Y 5$ activation was comparable to wild type, indicating that the 
bbx21-3D enhanced photomorphogenic phenotype was not associated with increased HY5 transcript levels (Supplemental Fig. S2A). However, we observed an increase in HY5 protein accumulation in $b b \times 21-3 D$ compared to wild type in response to UV-B (Fig. 1F). BBX21 ${ }^{\mathrm{P} 314 \mathrm{~L}}$ thus likely promotes HY5 accumulation at the post-transcriptional level. It is also of note that $B B X 21$ mRNA levels were not elevated in $b b \times 21-3 D$ compared to wild type (Supplemental Fig. S2B). Moreover, whereas UV-B-induced expression of RUP2 seemed unaffected by BBX21 (Supplemental Fig. S2C), several other UV-B marker genes such as EARLY LIGHT-INDUCIBLE PROTEIN2 (ELIP2), CHS, and FLAVANONE 3-HYDROXYLASE (F3H) showed lower gene activation in $b b \times 21-1$ compared to $b b \times 21-3 D$ (Supplemental Fig. S2D-F). Ultimately, $b b x 21-3 D$ showed constitutive acclimation to UV-B, as its photosynthetic capacity was less affected by a UV-B stress compared to the wild type (Fig. 1G). Altogether, these data support a positive regulatory role of BBX21 in the UVR8 signaling pathway.

As BBX21 promotes photomorphogenesis under visible light (Datta et al., 2007; Xu et al., 2016), we tested the $b b \times 21-3 D$ mutant in darkness and red and blue light. In darkness, $b b \times 21-1$ and $b b \times 21-3 D$ seedling phenotypes were comparable to wild type (Supplemental Fig. S3A,B). However, under both red and blue light, $b b \times 21-1$ indeed showed a reduced photomorphogenic phenotype relative to wild type, whereas bbx21-3D showed an enhanced photomorphogenic phenotype, including hypocotyl growth inhibition and anthocyanin accumulation (Supplemental Fig. S3C-H). Overall, the contrasting phenotypes of $b b \times 21-3 D$ and $b b \times 21-1$ support a positive regulatory role of BBX21 in photomorphogenic responses to both visible light and UV-B.

\section{BBX21 ${ }^{\mathrm{P} 14 \mathrm{~L}}$ function requires HY5 and shows increased activity in a both COP1-dependent} and -independent manner

The VP motif of BBX21 is located close to the C-terminus of the protein (residues 313/314 of the 331 amino acid protein; Supplemental Fig. S1A,B). This motif assumedly mediates the interaction with COP1, leading to polyubiquitination and proteasomal degradation of BBX21 during skotomorphogenesis (Xu et al., 2016; Job et al., 2018; Lau et al., 2019; Bursch et al., 2020). In agreement, mutation of the VP motif to Ala-Ala (AA) increased the post-translational stability of BBX21 (Bursch et al., 2020), C-terminal deletion including the VP motif mimicked the $b b x 21-3 D$ phenotype (Supplemental Fig. S1B,D), and the BBX21 ${ }^{\mathrm{P} 314 \mathrm{~L}}$ mutation resulted in hypermorphic phenotypes in all tested light conditions (Fig. 1 and Supplemental Fig. S3). 
We thus tested the interactions of BBX21 and BBX21 $1^{\mathrm{P} 314 \mathrm{~L}}$ with $\mathrm{HY} 5$ and the C-terminal WD40-domain of COP1 $\left(\mathrm{COP} 1^{\mathrm{C} 340}\right)$ in yeast two-hybrid $(\mathrm{Y} 2 \mathrm{H})$ assays. Whereas interaction of BBX21 with $\mathrm{COP} 1^{\mathrm{C} 340}$ was clearly detectable, BBX21 ${ }^{\mathrm{P} 314 \mathrm{~L}}$ interaction with $\mathrm{COP} 1^{\mathrm{C} 340}$ was impaired (Fig. 2A). Interestingly, by contrast, the interaction between BBX21 ${ }^{\mathrm{P} 314 \mathrm{~L}}$ and HY5 was strongly enhanced compared to the BBX21-HY5 interaction (Fig. 2A). The intrinsic transcriptional activities ("auto-activation") of BBX21 $1^{\mathrm{P} 314 \mathrm{~L}}$ and BBX21 fused to the LexA binding domain were comparable in the heterologous yeast system (Fig. 2B), making it unlikely that the $b b \times 21-3 D$ phenotype is due to intrinsically enhanced transcriptional activity of $\mathrm{BBX} 21^{\mathrm{P} 314 \mathrm{~L}}$. Nonetheless, the loss of interaction with COP1, and thus putative stabilization of BBX2 $1^{\mathrm{P} 314 \mathrm{~L}}$, and the enhanced $\mathrm{BBX} 21^{\mathrm{P} 314 \mathrm{~L}}-\mathrm{HY} 5$ interaction provide mutually non-exclusive mechanistic explanations for the $b b \times 21-3 D$ enhanced photomorphogenic phenotype.

We further compared the phenotypes of hy5-215 bbx21-3D (hy5 bbx21-3D) and cop 1-4 $b b \times 21-3 D($ copl bbx21-3D) double mutants to their corresponding single mutants. cop 1 bbx $1-3 D$ showed an enhanced constitutively photomorphogenic phenotype compared to copl (Fig. 2C-E; see also Fig. 3 and Supplemental Fig. S4). This supports the notion that BBX21 $1^{\mathrm{P} 14 \mathrm{~L}}$ may exhibit increased activity independent of its protein stabilization through removed COP1-mediated degradation. On the other hand, hy5 bbx21-3D showed no aberrant phenotype in darkness, as was observed for hy5 and bbx21-3D (Fig. 2C-E). However, under all light conditions tested, including UV-B (Fig. 3) and monochromatic red and blue light (Supplemental Fig. S4), hy5 was epistatic to $b b \times 21-3 D$, suggesting that the enhanced photomorphogenic phenotype of $b b \times 21-3 D$ is dependent on functional HY5.

\section{BBX21 and BBX22 are stabilized in response to UV-B}

The analysis of $b b \times 21$ null and hypermorphic mutants suggested a role for class IV BBX proteins, which include BBX20 and BBX22, in UV-B signaling. Whereas BBX20 was apparently not transcriptionally regulated by UV-B, $B B X 21$ transcript levels were slightly repressed by UV-B (Fig. 4A; see also Supplemental Fig. S2B). By contrast, within $1 \mathrm{~h}$ of UV-B exposure, $B B X 22$ expression induction was observed that was dependent on both UVR8 and HY5 (Fig. 4A,B). Beyond transcriptional regulation, BBX20, BBX21 and BBX22 proteins are post-translationally regulated by COP1 (Chang et al., 2011; Fan et al., 2012; Xu et al., 2016; Job et al., 2018). We thus tested BBX protein levels in lines expressing GFP-tagged BBX20, BBX21, and BBX22 under the 
195

196

197

198

199

200

201

202

203

204

205

206

207

208

209

210

211

212

213

214

215

216

217

218

219

220

221

222

223

224

225

constitutive cauliflower mosaic virus (CaMV) 35S promoter. GFP-BBX20 showed constitutive protein levels under UV-B (Fig. 4C), in agreement with the absence of a conserved VP motif in this protein (Supplemental Fig. S5). By contrast, transient stabilization of GFP-BBX21 was detectable after $1 \mathrm{~h}$ of UV-B (Fig. 4D). GFP-BBX22 was strongly stabilized in response to UV-B, with immunoblots revealing two specific bands (Fig. 4E), previously reported as representing fulllength and truncated forms of GFP-BBX22 (Chang et al., 2011). Full-length GFP-BBX22 levels peaked following approximately $3 \mathrm{~h}$ UV-B exposure, whereas the truncated GFP-BBX22 accumulated at least up to $9 \mathrm{~h} \mathrm{UV-B}$ treatment (Fig. 4E). Using antibodies specifically raised against a BBX22 $2^{199-213}$ peptide, we observed a similar pattern for endogenous BBX22 in wild type (Fig. 4F). We conclude that BBX21 and BBX22 are transiently stabilized under UV-B, consistent with the notion that their COP1-mediated degradation is relieved once UVR8 binds and represses COP1 activity.

\section{HY5 negatively regulates BBX21 and BBX22 accumulation under white light and UV-B}

To confirm that the observed BBX protein stabilization was indeed dependent on the UVR8 signaling pathway, we analyzed BBX21 and BBX22 levels in different genetic backgrounds. As expected, GFP-BBX21 and GFP-BBX22 protein stabilization under UV-B was absent in $u v r 8-12$ null mutants (Fig. 5A,B). This was confirmed for endogenous BBX22 (Fig. 5C). Conversely, in comparison to wild type, endogenous full-length and truncated BBX22 showed strongly enhanced levels in response to UV-B in uvr8-17D and rup1 rup2 (Fig. 5C), which are UV-B hypersensitive mutants due to impaired UVR8 inactivation through redimerization (Gruber et al., 2010; Heijde and Ulm, 2013; Podolec et al., 2021b). Interestingly, in the absence of HY5, strongly enhanced accumulation of GFP-BBX21 was observed before, after $1 \mathrm{~h}$ and particularly after $6 \mathrm{~h}$ of UV-B treatment (Fig. 5A). This contrasts with the very transient accumulation of GFP-BBX21 in wild type at $1 \mathrm{~h}$ of UV-B (Fig. 4D and Fig. 5A). These data suggest that HY5 negatively regulates $\mathrm{BBX} 21$ accumulation and is required for the transient nature of BBX21 stabilization under UV-B in wild type. A similar observation was made regarding BBX22 accumulation, where the absence of HY5 allowed the over-accumulation of BBX22 and shifted the peak of full-length BBX22 stabilization to later timepoints (Fig. 5B,D). BBX22 physically interacts with the bZIP domain of HY5 (Datta et al., 2008) and, together with BBX20 and BBX21, forms a transcriptional module that promotes photomorphogenesis (Bursch et al., 2020). We speculated that the repressive effect 
of HY5 on BBX22 levels may be explained by co-degradation of HY5 and BBX22 by COP1. However, this was not the case, as BBX22 was degraded normally in a hy5/Pro $35 \mathrm{~s}: \mathrm{HY} 5^{\Delta \mathrm{N} 77}$ line where the COP1-interacting N-terminus of HY5 is deleted and HY5 is thus constitutively stable (Fig. 5E) (Ang et al., 1998; Osterlund et al., 2000). On the other hand, BBX22 stabilization was strongly repressed in $b b \times 21-3 D$ and Pro35s:GFP-BBX21 lines (Fig. 5F). Overall, our data suggest that HY5 together with its BBX21 coactivator induce a repressor of BBX22 (and possibly BBX21) protein stability as part of a negative feedback loop included in the photomorphogenic program.

\section{BBX20, BBX21, and BBX22 promote UVR8- and HY5-dependent responses}

To determine whether BBX proteins contribute to UVR8- and HY5-dependent, UV-B-induced photomorphogenic responses, we tested the phenotypes of combinatorial mutants, including a bbx20 bbx21 bbx22 triple mutant. As shown previously (Favory et al., 2009; Tavridou et al., 2020), uvr8 mutant seedlings were strongly impaired in UV-B-induced hypocotyl growth inhibition, whereas hy5 showed longer hypocotyls under both -UV-B and +UV-B conditions, with reduced inhibition of hypocotyl elongation under UV-B (Fig. 6A,B and Supplemental Fig. S6A). The bbx20 bbx21 bbx22 triple mutant displayed longer hypocotyls in-UV-B conditions, similar to hy5, and an intermediate hypocotyl length between that of wild type and hy5 under UV-B (Fig. 6A,B and Supplemental Fig. S6A). These data indicate that BBX proteins partially regulate the HY5dependent inhibition of hypocotyl elongation under UV-B. On the other hand, anthocyanin accumulation was strongly compromised in the $b b \times 20 b b \times 21 b b \times 22$ triple mutant, resembling hy5 (Fig. 6C). Flavonol profiling by high performance thin layer chromatography (HPTLC) further revealed that bbx20bbx21bbx22 was strongly impaired in the UV-B-dependent accumulation of flavonol glycosides (Fig. 6D). It is of note that the hy5 bbx20 bbx21 bbx22 quadruple mutant showed a weak additive phenotype for both hypocotyl length and pigment accumulation, suggesting that a HY5-independent activity of BBX proteins exists in addition to the above described BBX-independent activity of HY5 (Fig. 6A-C). Related to pigment biosynthesis, UVB-induced CHS protein accumulation was impaired but not completely abolished in $b b \times 20 b b \times 21$ $b b x 22$, whereas it was undetectable in hy5 (Fig. 6E and Supplemental Fig. S6B), suggesting there are additional important regulators of HY5 activity besides BBX20, BBX21, and BBX22. In agreement with reduced CHS accumulation and impaired pigment biosynthesis, $C H S$ transcript 
did not accumulate to high levels under prolonged UV-B conditions in $b b \times 20 b b \times 21 b b \times 22$, similar to hy5 and unlike in UV-B-exposed wild-type seedlings (Fig. 6F).

\section{BBX20, BBX21, and BBX22 promote the expression of some genes under prolonged UV-B} but not their short-term induction after $U V-B$ exposure

BBX proteins regulate HY5 transcription (Xu et al., 2016; Bursch et al., 2020); however, both HY5 transcript and HY5 protein accumulation after UV-B exposure were not strongly affected in the bbx20 bbx21 bbx22 triple mutant (Fig. 7A,B). HY5-dependent induction of UV-B marker genes such as RUP2, CHS, and ELIP2 was only weakly, if at all, affected in $b b \times 20 b b \times 21 b b \times 22$ in response to UV-B (Fig. 7C-E). Overall, the induction of transcripts was largely independent of $\mathrm{BBX} 20, \mathrm{BBX} 21$, and $\mathrm{BBX} 22$, and the residual induction in bbx20 bbx21 bbx22 was HY5 dependent (Fig. 7B-E). This was in stark contrast to the impaired induction of $C H S$ after $4 \mathrm{~d}$ of continuous UV-B conditions (Fig. 6F). Thus, we checked the expression of other marker genes in continuous -UV-B and +UV-B conditions. Interestingly, in bbx20 bbx21 bbx22, F3H induction under prolonged UV-B was impaired as well, whereas MYB12 and ELIP2 were only partially affected (Fig. 8A-C). By contrast, whereas RUP1 and RUP2 UV-B induction maintained strong dependence on HY5, RUP1 and RUP2 transcripts accumulated normally under prolonged UV-B in $b b \times 20 b b \times 21 b b \times 22$ (Fig. 8D,E). In summary, our data suggest that BBX20, BBX21, and BBX22 redundantly promote pigment biosynthesis and the accumulation of some transcripts, such as $C H S$ and $F 3 H$, under prolonged UV-B. However, BBX20, BBX21, and BBX22 do not seem to regulate the early induction of HY5 and other UV-B marker genes immediately after UV-B exposure; and they play a partial role in the HY5-dependent inhibition of hypocotyl elongation. 


\section{DISCUSSION}

279 Upon UV-B perception, nuclear UVR8 promotes photomorphogenesis and acclimation by directly or indirectly modulating the activity of several transcriptional regulators (Favory et al., 2009; Qian et al., 2016; Yin et al., 2016; Liang et al., 2018; Yang et al., 2018; Liang et al., 2019; Qian et al., 2020; Podolec et al., 2021a). These include the bZIP transcriptional regulators HY5 and HYH that control the expression of many genes downstream of UVR8 and both the cryptochrome and phytochrome pathways (Ang et al., 1998; Holm et al., 2002; Ulm et al., 2004; Brown et al., 2005; Oravecz et al., 2006; Stracke et al., 2010; Burko et al., 2020). These photoreceptors inhibit the activity of the COP1/SPA E3 ubiquitin ligase complex, allowing accumulation of HY5 and HYH followed by induction of the photomorphogenic program in the light (Hoecker, 2017; Podolec and Ulm, 2018). Despite its important and broad function as transcriptional regulator, HY5 itself lacks a transcriptional activation domain (Ang et al., 1998; Stracke et al., 2010; Burko et al., 2020). Recently, BBX20, BBX21 and BBX22 were characterized as HY5 coregulators that provide transactivational activity, allowing transcriptional gene activation in response to both red light and the butenolide molecule karrikin (Bursch et al., 2020; Bursch et al., 2021).

Here, we uncovered a novel, hypermorphic $b b \times 21$ allele in a genetic screen for mutants with enhanced UV-B responses. BBX21 is a promoter of photomorphogenesis that is targeted by COP1 for degradation (Xu et al., 2016). We identified a $b b x 21-3 D$ mutant containing a P314L mutation, which functionally abolished the COP1 interaction domain of BBX21. This novel VP-mutated allele of a COP1 substrate allowed us to test the functional relevance of a VP motif in an endogenous context. The $b b x 21-3 D$ phenotype was largely consistent with that of previously published $B B X 21$ overexpression lines (Xu et al., 2016; Job et al., 2018), supporting the assumption that $\mathrm{BBX} 21^{\mathrm{P} 314 \mathrm{~L}}$ is post-translationally stabilized and thus present at elevated levels in the mutant background, as expected for a BBX21 VP mutant (Podolec and Ulm, 2018; Lau et al., 2019; Bursch et al., 2020). Unfortunately, we were not able to raise specific anti-BBX21 antibodies to probe directly for endogenous $\mathrm{BBX} 21^{\mathrm{P} 314 \mathrm{~L}}$ levels compared to wild-type BBX21. Interestingly, however, the $b b \times 21-3 D$ mutation enhanced the cop 1-4 mutant phenotype, although no interaction between BBX21 and COP1 is expected to occur in this genetic context since the corresponding COP $1^{\mathrm{N} 282}$ protein expressed in cop1-4 lacks the VP-interacting WD40 repeat domain (McNellis et al., 1994; Lau et al., 2019). These data suggest that BBX21 ${ }^{\mathrm{P} 314 \mathrm{~L}}$ also exhibits elevated activity 
copl and bbx21-3D could be due to a stronger HY5-BBX21 ${ }^{\mathrm{P} 314 \mathrm{~L}}$ interaction, as our yeast interaction data suggested, and thus increased activity of BBX21 as a HY5 coactivator (Bursch et al., 2020).

We found similarities but also interesting differences in the role of BBX proteins in UV-B signaling compared to that under visible light. Regarding hypocotyl elongation phenotype, hy5 and bbx20 bbx21 bbx22 were comparably elongated in constant white light devoid of UV-B, but differed under constant white light supplemented with UV-B, wherein BBX proteins accounted for only a part of the HY5-dependent response. Interestingly, and similar to our observations, the HY5-dependent hypocotyl response of seedlings during karrikin signaling relies only partially on BBX20 and BBX21 (Bursch et al., 2021). Furthermore, the BBX-dependent transcriptome accounted only for a fraction of HY5-regulated transcription under red light, suggesting that additional HY5 coregulators are indeed involved in some responses (Bursch et al., 2020). In contrast to the hypocotyl elongation phenotype, BBX proteins were crucial for UV-B-dependent biosynthesis of phenylpropanoid pigments (flavonols, anthocyanins). Intriguingly, when analyzing the molecular basis of this phenotype, we found that gene expression involved in phenylpropanoid biosynthesis $(C H S, F 3 H)$ was impaired under prolonged and constant UV-B, but the induction of early UV-B marker genes within 6 h of UV-B exposure was almost completely intact. Similarly, CHS protein accumulation after UV-B exposure was decreased but not abolished in the $b b \times 20$ $b b \times 21 b b \times 22$ triple mutant. These data suggest that early and transient HY 5 responses to UV-B are $\mathrm{BBX} 20, \mathrm{BBX} 21$, and BBX22 independent, whereas for long-term responses such as pigment biosynthesis, HY5 relies on these BBX proteins as transcriptional coactivators. It is of note that the long-term accumulation of some transcripts, such as RUP1 and RUP2, was also BBXindependent.

BBX21 and BBX22 were post-transcriptionally stabilized under UV-B in a UVR8-dependent manner. This is consistent with their status as COP1 substrates (Chang et al., 2011; Xu et al., 2016; this work) and how active UVR8 directly inhibits COP1 activity by competitively blocking the COP1 substrate binding site (Lau et al., 2019; Podolec et al., 2021a). Consistent with previous literature on visible light responses (Chang et al., 2011; Xu et al., 2016; Job et al., 2018), we observed a transient stabilization of BBX21 and BBX22, with protein levels decreasing after a few hours of UV-B exposure. This transient stabilization could be linked to attenuation of UVR8 signaling through the RUP1- and RUP2-mediated negative feedback loop (Gruber et al., 2010; 
340 Heijde and Ulm, 2013; Ren et al., 2019; Podolec et al., 2021b), as supported by extended BBX22

341 accumulation in $u v r 8-17 D$ and rup1 rup2 backgrounds. Moreover, our data indicate the existence

342 of an additional negative regulator of BBX21 and BBX22 stability. This repressor seems to be

343 linked to HY5 activity, as suggested by the overaccumulation of BBX21 and BBX22 in hy5

344 backgrounds and as already reported for BBX22 under visible light (Chang et al., 2011).

345 Conversely, plant lines with enhanced photomorphogenesis (such as bbx21-3D and BBX21

346 overexpression lines) show lower levels of BBX22 accumulation. Collectively, our data point to

347 an uncharacterized negative feedback mechanism that attenuates photomorphogenesis induced by

348 the HY5/BBX transcriptional module.

349 We conclude that BBX20, BBX21, and BBX22 play important roles as HY5 coactivators in

350 inducing UV-B responses such as pigment biosynthesis, and to a lesser degree inhibition of

351 hypocotyl elongation, mainly in constant UV-B conditions (for our working model, see Fig. 8F).

352 Short-term responses on the other hand (few hours after UV-B exposure) seem to be mostly

353 independent of these BBX proteins, as seen by the normal transcriptional induction of HY5-

354 dependent UV-B marker genes in $b b \times 20 b b \times 21 b b \times 22$. This further indicates the existence of a

355 specific HY5 coactivator, or multiple thereof, under UV-B, the activation of which is likely crucial

356 for early gene activation upon UV-B reception and signaling by the UVR8 photoreceptor. Whether

357 COP1 regulates further HY5 coregulators and whether they are members of the large BBX family

358 remains to be determined. 


\section{MATERIALS AND METHODS}

360

361

362

363

364

365

366

367

368

369

370

371

372

373

374

375

376

377

378

379

380

381

382

383

384

385

386

387

388

389

\section{Plant materials}

All lines used in this study are in the Arabidopsis (Arabidopsis thaliana) Columbia (Col-0) background. The following lines have been described previously: rup1 rup2 (Gruber et al., 2010), cop1-4 (Deng et al., 1992), uvr8-12, uvr8-17D (Podolec et al., 2021b), hy5-215 (Oyama et al., 1997), hy5-215 hyh (Zoulias et al., 2020), bbx21-1 (Datta et al., 2007), bbx22-1 (Chang et al., 2008), bbx20-1, bbx21-1 bbx22-1, bbx20-1 bbx21-1 bbx22-1, hy5-215 bbx20-1 bbx21-1 bbx22-1, Col-0/Pro $35 s: G F P-B B X 20 ~ \# 1$ and \#2, hy5-215/Pro $35 s: H Y 5^{\Delta N 77} \# 27, h y 5-215 /$ Pro $_{35 s}: G F P-B B X 21$ \#2 (Bursch et al., 2020), bbx20-1 bbx21-1 (Bursch et al., 2021), Col-0/Pro35s:GFP-BBX21 \#1 and \#2, hy5-215/Pro $35 s: H Y 5$ \#15 (Job et al., 2018). Pro35s:GFP-BBX22 lines \#1 and \#2 were generated in the Col-0 background using a pB7WGF2 vector (Karimi et al., 2002) in which the $B B X 22$ CDS was inserted after cloning into pDONR221 (primers BBX22_attB1_Fw and BBX22_attB2_Rv, Table S1) using Gateway technology (ThermoFisher). bbx21-3D was identified in a forward genetic screen based on hypocotyl length under UV-B of an EMS mutagenized Col-0 population (Podolec et al., 2021b), and was backcrossed three times. Combinatorial mutant lines bbx20-1 bbx22-1, hy5-215 bbx21-3D, cop1-4 bbx21-3D, uvr8-12/Pro35s:GFP-BBX21 \#2, uvr812/Pro $35 \mathrm{~s}: G F P-B B X 22$ \#1, and hy5-215/Pro $35 \mathrm{~s}:$ GFP-BBX22 \#1 were generated by crossing and genotyped by PCR and sequencing.

\section{Generation of $B B X 21$ CRISPR/Cas9-mutated lines}

The CRISPR/Cas9 system was used to delete the $B B X 21 \mathrm{C}$-terminus in wild type and to knockout $B B X 21$ in wild type and $b b \times 21-3 D$. The sgRNA directed against the $B B X 21$ sequence were inserted into the pHEE401E vector (Wang et al., 2015) using overlapping complementary oligos (Supplemental Table S1). Arabidopsis plants were then transformed using the floral-dip method (Clough and Bent, 1998). Several independent transgenic events were selected and the $B B X 21$ locus was sequenced in T2 to identify lines with desired mutations.

\section{Growth conditions and light treatments}

Seeds were surface sterilized using chlorine gas or ethanol and sown on $1 / 2$ MS (Duchefa) agar medium supplemented with $1 \%(\mathrm{w} / \mathrm{v})$ sucrose (except for experiments in monochromatic red/blue light, which were done on media without sucrose). Plates were left for $2 \mathrm{~d}$ in the dark at $4^{\circ} \mathrm{C}$ for 
390

391

392

393

394

395

396

397

398

399

400

401

402

403

404

405

406

407

408

409

410

411

412

413

414

415

416

417

418

419

420

stratification. For growth in darkness, plates were exposed for $6 \mathrm{~h}$ to $\sim 60 \mu \mathrm{mol} \mathrm{m} \mathrm{m}^{-2} \mathrm{~s}^{-1}$ of white light and then transferred to darkness at $22^{\circ} \mathrm{C}$ for $4 \mathrm{~d}$. For light treatments, plates were grown for $4 \mathrm{~d}$ at $22^{\circ} \mathrm{C}$ in the following light conditions: weak white light $\left(3.6 \mu \mathrm{mol} \mathrm{m} \mathrm{m}^{-2} \mathrm{~s}^{-1}\right.$, Osram L18W/30 tubes) supplemented or not with narrowband UV-B $\left(1.5 \mu \mathrm{mol} \mathrm{m}^{-2} \mathrm{~s}^{-1}\right.$, Philips TL20W/01RS tubes), monochromatic red light $\left(150 \mu \mathrm{mol} \mathrm{m} \mathrm{m}^{-2} \mathrm{~s}^{-1}\right.$, floraLEDs in CLF Plant Climatics cabinet), monochromatic blue light $\left(50 \mu \mathrm{mol} \mathrm{m} \mathrm{m}^{-2} \mathrm{~s}^{-1}\right.$, floraLEDs in CLF Plant Climatics cabinet). For UVB stress treatment, plates were irradiated with broadband UV-B (21 $\mu \mathrm{mol} \mathrm{m} \mathrm{m}^{-2} \mathrm{~s}^{-1}$, Philips TL20W/12RS tubes).

\section{Hypocotyl length measurements}

Hypocotyl length was determined as described previously (Podolec et al., 2021b). In short, seedlings were grown for $4 \mathrm{~d}$ in the appropriate condition and approximately 60 seedlings for each genotype/condition were aligned on an agar plate and scanned. Individual hypocotyls were measured using the NeuronJ plugin of ImageJ (Meijering et al., 2004).

\section{Extraction and quantification of anthocyanins}

Anthocyanins were quantified as described previously (Yin et al., 2012). Seedlings were grown for $4 \mathrm{~d}$ in the appropriate condition and approximately $50 \mathrm{mg}$ of seedlings were collected for each genotype and condition. Samples were frozen in liquid nitrogen, ground, and $250 \mu 1$ of extraction buffer $(99 \%[\mathrm{v} / \mathrm{v}]$ methanol, $1 \%[\mathrm{v} / \mathrm{v}] \mathrm{HCl})$ was added to the samples. Extraction was performed for at least $1 \mathrm{~h}$ by incubating the samples on a rotary shaker at $4^{\circ} \mathrm{C}$. After centrifugation for $5 \mathrm{~min}$, the absorbance of $150 \mu \mathrm{l}$ of the clear supernatant was measured at $530 \mathrm{~nm}$ and $655 \mathrm{~nm}$. The following formula was used to calculate the relative quantity of anthocyanins: $\left(\mathrm{A}_{530}-0.25 * \mathrm{~A}_{655}\right)$ / seedling mass (in mg).

\section{Extraction and visualization of flavonoids}

High-performance thin layer chromatography (HPTLC) was used to analyze the flavonol profile as described previously (Podolec et al., 2021b). In short, exactly $50 \mathrm{mg}$ of seedlings were collected for each genotype and condition, samples were frozen and then ground. $100 \mu 1$ of extraction buffer $\left(80 \%[\mathrm{v} / \mathrm{v}]\right.$ methanol) was added per sample, which were then incubated for $10 \mathrm{~min}$ at $70^{\circ} \mathrm{C}$ on a shaker. After a 5 min centrifugation at $14,000 \mathrm{rpm}$, supernatants were collected and $10 \mu 1$ was 
421 loaded on silica HPTLC plates. The extracts were then separated for approximately 45 min using 422 a mobile phase ( $5 \mathrm{ml}$ ethyl acetate, $600 \mu \mathrm{l}$ formic acid, $600 \mu \mathrm{l}$ acetic acid glacial, $1.3 \mathrm{ml}$ water). 423 After migration, the plate was dried and sprayed with 1\% (w/v) diphenylboric acid 2424 aminoethylester (DPBA, Roth) solution in $80 \%(\mathrm{v} / \mathrm{v})$ methanol. The plate was exposed under a 365-nm UV-A lamp to reveal the flavonoid profile.

\section{Measurements of photosynthetic efficiency}

428 Maximum quantum efficiency of photosystem II was measured after dark-adapting plants for 5 min using a Fluorcam (Photon Systems Instruments) with blue (470 nm) LEDs, and was calculated as $\mathrm{Fv} / \mathrm{Fm}=(\mathrm{Fm}-\mathrm{Fo}) / \mathrm{Fm}$, where $\mathrm{Fm}$ is the maximal fluorescence and Fo the minimal fluorescence in the dark-adapted state.

\section{Protein extraction and immunoblot assays}

434 For all immunoblot assays that include (GFP-)BBX levels, a previously described buffer (Job et al., 2018) was used: $50 \mathrm{mM}$ Tris- $\mathrm{HCl} \mathrm{pH}$ 7.5, $150 \mathrm{mM} \mathrm{NaCl}, 1 \%(\mathrm{w} / \mathrm{v})$ sodium deoxycholate, 0.5\% (v/v) Triton X100, 1 mM DTT, $50 \mu \mathrm{M}$ MG132 (Sigma), $50 \mu \mathrm{M}$ ALLN (VWR), and $50 \mu \mathrm{M}$

437 Protease Inhibitor Cocktail (Sigma). For HY5 immunoblots, a previously described buffer 438 (Oravecz et al., 2006) was used: 0.1 M Tris-HCl pH 8.0, $50 \mu \mathrm{M}$ EDTA, $0.25 \mathrm{M} \mathrm{NaCl,} \mathrm{0.7 \% (w/v)}$ SDS, $10 \mathrm{mM} \mathrm{NaF}, 15 \mathrm{mM} \beta$-glycerolphosphate, $15 \mathrm{mM}$ p-nitrophenyl phosphate, a Complete EDTA-free Protease Inhibitor Cocktail tablet (Roche), and $1 \mathrm{mM}$ DTT. For CHS immunoblot assays, a previously described phosphate buffer (Arongaus et al., 2018) was used: $50 \mathrm{mM} \mathrm{Na-}$ phosphate $\mathrm{pH}$ 7.4, $150 \mathrm{mM} \mathrm{NaCl}, 10 \%$ (v/v) glycerol, $5 \mathrm{mM}$ EDTA, 0.1\% (v/v) Triton X-100, 1 mM DTT, 2 mM Na 3 VO4, 2 mM NaF, 1\% (v/v) Protease Inhibitor Cocktail (Sigma), and $50 \mu \mathrm{M}$ of MG132 (Sigma).

In all cases except for HY5 immunoblots, samples were harvested, frozen, ground, and mixed

447 were collected and protein concentration was determined using the Bradford-based Bio-Rad protein assay (Bio-Rad). Samples were then denatured and separated using SDS-PAGE. Proteins were transferred on PVDF membranes (Roth) for 7 min at $20 \mathrm{~V}$ using the iBlot dry blotting system

450 (Thermo Fisher Scientific), before blocking in TBS-T with milk. 
For HY5 immunoblots, proteins were extracted as described above and wet-transferred 452 (transfer buffer: $25 \mathrm{mM}$ Tris; $192 \mathrm{mM}$ glycine; 20\% [v/v] ethanol) on PVDF membranes (Roth).

453 Membranes were rinsed once with water, once with TBS-T, then dried for 15 min in a speed-vac 454 at $65^{\circ} \mathrm{C}$ and kept for $5 \mathrm{~d}$ at room temperature in the dark. Subsequent incubation steps were 455 performed in TBS-T without milk.

456 The following primary antibodies were used: anti-CHS (sc-12620, Santa Cruz 457 Biotechnology), anti-GFP (Living Colors ${ }^{\circledR}$ A.v. Monoclonal Antibody, JL-8; Clontech), anti-HY5 458 (Oravecz et al., 2006), anti-actin (A0480, Sigma-Aldrich), anti-BBX22(199-213) (Eurogentec, raised 459 in rabbits against the peptide C+DQSYEYMENNGSSKT and affinity purified). Corresponding 460 horseradish peroxidase-conjugated anti-rabbit (for anti-HY5 and anti-BBX22), anti-mouse (for 461 anti-GFP and anti-actin), and anti-goat (for anti-CHS) immunoglobulins (Dako) were used as 462 secondary antibodies. Signal was revealed using the ECL Select Western Blotting Detection 463 Reagent (GE Healthcare) and analyzed using an Amersham Imager 680 camera system (GE 464 Healthcare).

465

\section{Yeast two-hybrid assays}

467 Yeast two-hybrid assays were performed after transforming the L40 strain (Vojtek and Hollenberg, 468 1995) using the lithium-acetate protocol (Gietz, 2014). $B B X 21$ and $B B X 21^{P 314 L}$ coding sequences were inserted in frame with the Gal4 activation domain (Gal4-AD) into pGADT7-GW (Marrocco et al., 2006) and $C O P 1^{C 340}$ and $H Y 5$ were inserted in frame with the LexA DNA binding domain (LexA-DB) into pBTM116-D9-GW (Stelzl et al., 2005) using Gateway cloning. To test for

473 LexA-DB into pBTM116-D9-GW (Stelzl et al., 2005) using Gateway cloning. Multiple colonies 474 from each transformation were mixed, spotted, and grown on vector-selection medium (SD/-Trp/475 Leu; SD/-Trp for testing transcriptional activation potential). For growth assays, -His selective 476 medium for interactions (SD/-Trp/-Leu/-His, Formedium) was used. For quantitative $\beta$ 477 galactosidase assays, yeast cells were grown for $2 \mathrm{~d}$ on vector-selection plates, collected and the 478 enzymatic assay was performed using red- $\beta$-D-galactopyranoside (CPRG, Roche Applied 479 Science) as substrate as described (Yeast Protocols Handbook, Clontech).

\section{Gene expression analysis}


To determine transcript levels by reverse transcription quantitative PCR (RT-qPCR), RNA was extracted using the ReliaPrep RNA Tissue Miniprep System kit (Promega) and treated with DNase according to the manufacturer's instructions. cDNA synthesis was performed using the TaqMan reverse transcription kit (Applied Biosystems), with a 1:1 mix of oligo-dT and random hexamer primers. The qPCR reaction was performed using the PowerUp SYBR Green Master Mix reagents (Applied Biosystems) on a QuantStudio ${ }^{\text {TM }} 5$ System (Thermo Fisher Scientific) following the recommended protocol, using primers listed in Supplemental Table S1. The $\Delta \Delta \mathrm{Ct}$ method (Livak and Schmittgen, 2001) was used to calculate expression values, with $P P 2 A$ as a reference gene. Each experiment was done by combining 3-6 independently made biological replicates. All expression values were normalized within each biological replicate against the untreated wild type that was set to 1 .

\section{Statistical analysis}

Statistical analyses were done using the GraphPad Prism software (v. 9.2.0). One- or two-way ANOVAs were performed on log-transformed or non-transformed data, followed by Tukey's test for multiple comparisons to distinguish statistically different groups $(\mathrm{P}>0.05)$ using different letters. For the analysis of Fv/Fm (Fig. 1G), a repeated measures ANOVA was used. For pairwise comparisons (Fig. 4A), unpaired two-tailed $t$-tests were used and $\mathrm{P}$ values were represented.

\section{Accession numbers}

Sequence data from this work can be found in the Arabidopsis Genome Initiative or GenBank/EMBL databases under the following accession numbers: AT4G39070 (BBX20), AT1G75540 (BBX21), AT1G78600 (BBX22), AT5G13930 (CHS), AT3G51240 (F3H), AT2G32950 (COP1), AT5G11260 (HY5), AT3G17609 (HYH), AT2G47460 (MYB12), AT4G14690 (ELIP2), AT5G52250 (RUP1), AT5G23730 (RUP2), AT5G63860 (UVR8).

\section{Supplemental Data}

Supplemental Figure S1. The $b b \times 21-3 D$ phenotype is dependent on functional $B B X 21$, and can be recapitulated by deleting the C-terminal 25 amino acids that include the VP motif.

Supplemental Figure S2. BBX21 promotes the expression of some marker genes in response to UV-B. 
513 Supplemental Figure S3. $b b \times 21-3 D$ phenotype in darkness and monochromatic red and blue

514 light.

515 Supplemental Figure S4. Genetic relationship between $b b x 21-3 D$ and cop 1 and $h y 5$ in

516 monochromatic red and blue light conditions.

517 Supplemental Figure S5. Conservation of putative VP motifs in BBX20, BBX21, and BBX22.

518 Supplemental Figure S6. Genetic redundancy between BBX20, BBX21, and BBX22.

519 Supplemental Table S1. Primers used in this study.

520

521 ACKNOWLEDGEMENTS

522 We would like to thank Emilie Demarsy and Michel Goldschmidt-Clermont for critically reading

523 the manuscript. This work was supported by the University of Geneva, the Swiss National Science

524 Foundation (grant 31003A_175774 to R.U.), and an iGE3 PhD salary award (to R.P.). H.J. was

525 supported by the German Research Foundation (project number 320656366).

526

527 AUTHOR CONTRIBUTIONS

528 R.P. and R.U. designed the research; T.B.W., R.P., and M.L. performed the experiments; H.J. 529 contributed new tools; T.B.W., R.P., and R.U. analyzed the data and wrote the paper. All authors 530 reviewed and approved the submitted manuscript. 


\section{REFERENCES}

Ang, L.H., Chattopadhyay, S., Wei, N., Oyama, T., Okada, K., Batschauer, A., and Deng, X.W. (1998). Molecular interaction between COP1 and HY5 defines a regulatory switch for light control of Arabidopsis development. Mol Cell 1, 213-222.

Arongaus, A.B., Chen, S., Pireyre, M., Glockner, N., Galvao, V.C., Albert, A., Winkler, J.B., Fankhauser, C., Harter, K., and UIm, R. (2018). Arabidopsis RUP2 represses UVR8-mediated flowering in noninductive photoperiods. Genes Dev 32, 1332-1343.

Bai, S., Saito, T., Honda, C., Hatsuyama, Y., Ito, A., and Moriguchi, T. (2014). An apple B-box protein, MdCOL11, is involved in UV-B- and temperature-induced anthocyanin biosynthesis. Planta 240, 1051-1062.

Binkert, M., Kozma-Bognar, L., Terecskei, K., De Veylder, L., Nagy, F., and Ulm, R. (2014). UV-Bresponsive association of the Arabidopsis bZIP transcription factor ELONGATED HYPOCOTYL5 with target genes, including its own promoter. Plant Cell 26, 4200-4213.

Brown, B.A., and Jenkins, G.I. (2008). UV-B signaling pathways with different fluence-rate response profiles are distinguished in mature Arabidopsis leaf tissue by requirement for UVR8, HY5, and HYH. Plant Physiol 146, 576-588.

Brown, B.A., Cloix, C., Jiang, G.H., Kaiserli, E., Herzyk, P., Kliebenstein, D.J., and Jenkins, G.I. (2005). A UV-B-specific signaling component orchestrates plant UV protection. Proc Natl Acad Sci USA 102, 18225-18230.

Burko, Y., Seluzicki, A., Zander, M., Pedmale, U.V., Ecker, J.R., and Chory, J. (2020). Chimeric activators and repressors define HY5 activity and reveal a light-regulated feedback mechanism. Plant Cell 32, 967-983.

Bursch, K., Niemann, E.T., Nelson, D.C., and Johansson, H. (2021). Karrikins control seedling photomorphogenesis and anthocyanin biosynthesis through a HY5-BBX transcriptional module. Plant J 107, 1346-1362.

Bursch, K., Toledo-Ortiz, G., Pireyre, M., Lohr, M., Braatz, C., and Johansson, H. (2020). Identification of BBX proteins as rate-limiting cofactors of HY5. Nat Plants 6, 921-928.

Chang, C.-S.J., Maloof, J.N., and Wu, S.-H. (2011). COP1-mediated degradation of BBX22/LZF1 optimizes seedling development in Arabidopsis. Plant Physiol 156, 228-239.

Chang, C.-S.J., Li, Y.-H., Chen, L.-T., Chen, W.-C., Hsieh, W.-P., Shin, J., Jane, W.-N., Chou, S.-J., Choi, G., Hu, J.-M., Somerville, S., and Wu, S.-H. (2008). LZF1, a HY5-regulated transcriptional factor, functions in Arabidopsis de-etiolation. Plant J 54, 205-219.

Cloix, C., Kaiserli, E., Heilmann, M., Baxter, K.J., Brown, B.A., O'Hara, A., Smith, B.O., Christie, J.M., and Jenkins, G.I. (2012). C-terminal region of the UV-B photoreceptor UVR8 initiates signaling through interaction with the COP1 protein. Proc Natl Acad Sci USA 109, 16366-16370.

Clough, S.J., and Bent, A.F. (1998). Floral dip: a simplified method for Agrobacterium-mediated transformation of Arabidopsis thaliana. Plant J 16, 735-743.

Crocco, C.D., Locascio, A., Escudero, C.M., Alabadí, D., Blázquez, M.A., and Botto, J.F. (2015). The transcriptional regulator BBX24 impairs DELLA activity to promote shade avoidance in Arabidopsis thaliana. Nat Commun 6, 6202.

Datta, S., Hettiarachchi, C., Johansson, H., and Holm, M. (2007). SALT TOLERANCE HOMOLOG2, a B-box protein in Arabidopsis that activates transcription and positively regulates light-mediated development. Plant Cell 19, 3242-3255.

Datta, S., Johansson, H., Hettiarachchi, C., Irigoyen, M.a.L., Desai, M., Rubio, V., and Holm, M. (2008). LZF1/SALT TOLERANCE HOMOLOG3, an Arabidopsis B-box protein involved in light-dependent development and gene expression, undergoes COP1-mediated ubiquitination. Plant Cell 20, 2324-2338. 
Demarsy, E., Goldschmidt-Clermont, M., and UIm, R. (2018). Coping with 'dark sides of the sun' through photoreceptor signaling. Trends Plant Sci 23, 260-271.

Deng, X.-W., Matsui, M., Wei, N., Wagner, D., Chu, A.M., Feldmann, K.A., and Quail, P.H. (1992). COP1, an Arabidopsis regulatory gene, encodes a protein with both a zinc-binding motif and a $G \beta$ homologous domain. Cell 71, 791-801.

Fan, X.-Y., Sun, Y., Cao, D.-M., Bai, M.-Y., Luo, X.-M., Yang, H.-J., Wei, C.-Q., Zhu, S.-W., Sun, Y., Chong, K., and Wang, Z.-Y. (2012). BZS1, a B-box protein, promotes photomorphogenesis downstream of both brassinosteroid and light signaling pathways. Mol Plant 5, 591-600.

Fang, H., Dong, Y., Yue, X., Hu, J., Jiang, S., Xu, H., Wang, Y., Su, M., Zhang, J., Zhang, Z., Wang, N., and Chen, $X$. (2019). The B-box zinc finger protein MdBBX20 integrates anthocyanin accumulation in response to ultraviolet radiation and low temperature. Plant Cell Environ 42, 2090-2104.

Favory, J.J., Stec, A., Gruber, H., Rizzini, L., Oravecz, A., Funk, M., Albert, A., Cloix, C., Jenkins, G.I., Oakeley, E.J., Seidlitz, H.K., Nagy, F., and UIm, R. (2009). Interaction of COP1 and UVR8 regulates UV-B-induced photomorphogenesis and stress acclimation in Arabidopsis. EMBO J 28, 591-601.

Galvao, V.C., and Fankhauser, C. (2015). Sensing the light environment in plants: photoreceptors and early signaling steps. Curr Opin Neurobiol 34, 46-53.

Gangappa, S.N., Crocco, C.D., Johansson, H., Datta, S., Hettiarachchi, C., Holm, M., and Botto, J.F. (2013). The Arabidopsis B-BOX protein BBX25 interacts with HY5, negatively regulating BBX22 expression to suppress seedling photomorphogenesis. Plant Cell 25, 1243-1257.

Gietz, R.D. (2014). Yeast transformation by the LiAc/SS carrier DNA/PEG method. In Yeast Genetics: Methods and Protocols, J.S. Smith and D.J. Burke, eds (New York, NY: Springer New York), pp. 112.

Gruber, H., Heijde, M., Heller, W., Albert, A., Seidlitz, H.K., and Ulm, R. (2010). Negative feedback regulation of UV-B-induced photomorphogenesis and stress acclimation in Arabidopsis. Proc Natl Acad Sci USA 107, 20132-20137.

Heijde, M., and UIm, R. (2013). Reversion of the Arabidopsis UV-B photoreceptor UVR8 to the homodimeric ground state. Proc Natl Acad Sci USA 110, 1113-1118.

Hoecker, U. (2017). The activities of the E3 ubiquitin ligase COP1/SPA, a key repressor in light signaling. Curr Opin Plant Biol 37, 63-69.

Holm, M., Hardtke, C.S., Gaudet, R., and Deng, X.-W. (2001). Identification of a structural motif that confers specific interaction with the WD40 repeat domain of Arabidopsis COP1. EMBO J 20, 118127.

Holm, M., Ma, L.G., Qu, L.J., and Deng, X.W. (2002). Two interacting bZIP proteins are direct targets of COP1-mediated control of light-dependent gene expression in Arabidopsis. Genes Dev 16, 12471259.

Huang, X., Ouyang, X., Yang, P., Lau, O.S., Chen, L., Wei, N., and Deng, X.W. (2013). Conversion from CUL4-based COP1-SPA E3 apparatus to UVR8-COP1-SPA complexes underlies a distinct biochemical function of COP1 under UV-B. Proc Natl Acad Sci USA 110, 16669-16674.

Huang, X., Ouyang, X., Yang, P., Lau, O.S., Li, G., Li, J., Chen, H., and Deng, X.W. (2012). Arabidopsis FHY3 and HY5 positively mediate induction of COP1 transcription in response to photomorphogenic UV-B light. Plant Cell 24, 4590-4606.

Indorf, M., Cordero, J., Neuhaus, G., and Rodríguez-Franco, M. (2007). Salt tolerance (STO), a stressrelated protein, has a major role in light signalling. Plant J 51, 563-574.

Jiang, L., Wang, Y., Li, Q.F., Bjorn, L.O., He, J.X., and Li, S.S. (2012). Arabidopsis STO/BBX24 negatively regulates UV-B signaling by interacting with COP1 and repressing HY5 transcriptional activity. Cell Res 22, 1046-1057. 
Job, N., Yadukrishnan, P., Bursch, K., Datta, S., and Johansson, H. (2018). Two B-box proteins regulate photomorphogenesis by oppositely modulating HY5 through their diverse C-terminal domains. Plant Physiol 176, 2963-2976.

Kami, C., Lorrain, S., Hornitschek, P., and Fankhauser, C. (2010). Light-regulated plant growth and development. Curr Top Dev Biol 91, 29-66.

Karimi, M., Inzé, D., and Depicker, A. (2002). GATEWAY'⿳一 vectors for Agrobacterium-mediated plant transformation. Trends Plant Sci 7, 193-195.

Kliebenstein, D.J., Lim, J.E., Landry, L.G., and Last, R.L. (2002). Arabidopsis UVR8 regulates ultraviolet-B signal transduction and tolerance and contains sequence similarity to human regulator of chromatin condensation 1. Plant Physiol 130, 234-243.

Lau, K., Podolec, R., Chappuis, R., Ulm, R., and Hothorn, M. (2019). Plant photoreceptors and their signaling components compete for COP1 binding via VP peptide motifs. EMBO J 38, e102140.

Liang, T., Yang, Y., and Liu, H. (2019). Signal transduction mediated by the plant UV-B photoreceptor UVR8. New Phytol 221, 1247-1252.

Liang, T., Mei, S., Shi, C., Yang, Y., Peng, Y., Ma, L., Wang, F., Li, X., Huang, X., Yin, Y., and Liu, H. (2018). UVR8 interacts with BES1 and BIM1 to regulate transcription and photomorphogenesis in Arabidopsis. Dev Cell 44, 512-523

Livak, K.J., and Schmittgen, T.D. (2001). Analysis of relative gene expression data using real-time quantitative PCR and the 2- $\triangle \Delta C T$ method. Methods 25, 402-408.

Marrocco, K., Zhou, Y., Bury, E., Dieterle, M., Funk, M., Genschik, P., Krenz, M., Stolpe, T., and Kretsch, T. (2006). Functional analysis of EID1, an F-box protein involved in phytochrome A-dependent light signal transduction. Plant J 45, 423-438.

McNellis, T.W., von Arnim, A.G., Araki, T., Komeda, Y., Misera, S., and Deng, X.W. (1994). Genetic and molecular analysis of an allelic series of cop1 mutants suggests functional roles for the multiple protein domains. Plant Cell 6, 487-500.

Meijering, E., Jacob, M., Sarria, J.-C.F., Steiner, P., Hirling, H., and Unser, M. (2004). Design and validation of a tool for neurite tracing and analysis in fluorescence microscopy images. Cytometry Part A 58A, 167-176.

Oravecz, A., Baumann, A., Mate, Z., Brzezinska, A., Molinier, J., Oakeley, E.J., Adam, E., Schafer, E., Nagy, F., and UIm, R. (2006). CONSTITUTIVELY PHOTOMORPHOGENIC1 is required for the UV-B response in Arabidopsis. Plant Cell 18, 1975-1990.

Osterlund, M.T., Hardtke, C.S., Wei, N., and Deng, X.W. (2000). Targeted destabilization of HY5 during light-regulated development of Arabidopsis. Nature 405, 462-466.

Oyama, T., Shimura, Y., and Okada, K. (1997). The Arabidopsis HY5 gene encodes a bZIP protein that regulates stimulus-induced development of root and hypocotyl. Genes Dev 11, 2983-2995.

Podolec, R., and UIm, R. (2018). Photoreceptor-mediated regulation of the COP1/SPA E3 ubiquitin ligase. Curr Opin Plant Biol 45, 18-25.

Podolec, R., Demarsy, E., and UIm, R. (2021a). Perception and signaling of ultraviolet-B radiation in plants. Annu Rev Plant Biol 72, 793-822.

Podolec, R., Lau, K., Wagnon, T.B., Hothorn, M., and Ulm, R. (2021b). A constitutively monomeric UVR8 photoreceptor confers enhanced UV-B photomorphogenesis. Proc Natl Acad Sci USA 118, e2017284118.

Qian, C., Mao, W., Liu, Y., Ren, H., Lau, O.S., Ouyang, X., and Huang, X. (2016). Dual-source nuclear monomers of UV-B light receptor direct photomorphogenesis in Arabidopsis. Mol Plant 9, 16711674.

Qian, C., Chen, Z., Liu, Q., Mao, W., Chen, Y., Tian, W., Liu, Y., Han, J., Ouyang, X., and Huang, X. (2020). Coordinated transcriptional regulation by the UV-B photoreceptor and multiple transcription factors for plant UV-B responses. Mol Plant 13, 777-792. 
Rai, N., Morales, L.O., and Aphalo, P.J. (2021). Perception of solar UV radiation by plants: photoreceptors and mechanisms. Plant Physiol 186, 1382-1396.

Ren, H., Han, J., Yang, P., Mao, W., Liu, X., Qiu, L., Qian, C., Liu, Y., Chen, Z., Ouyang, X., Chen, X., Deng, X.W., and Huang, X. (2019). Two E3 ligases antagonistically regulate the UV-B response in Arabidopsis. Proc Natl Acad Sci USA 116, 4722-4731.

Rizzini, L., Favory, J.J., Cloix, C., Faggionato, D., O'Hara, A., Kaiserli, E., Baumeister, R., Schafer, E., Nagy, F., Jenkins, G.I., and Ulm, R. (2011). Perception of UV-B by the Arabidopsis UVR8 protein. Science 332, 103-106.

Stelzl, U., Worm, U., Lalowski, M., Haenig, C., Brembeck, F.H., Goehler, H., Stroedicke, M., Zenkner, M., Schoenherr, A., Koeppen, S., Timm, J., Mintzlaff, S., Abraham, C., Bock, N., Kietzmann, S., Goedde, A., Toksöz, E., Droege, A., Krobitsch, S., Korn, B., Birchmeier, W., Lehrach, H., and Wanker, E.E. (2005). A human protein-protein interaction network: a resource for annotating the proteome. Cell 122, 957-968.

Stracke, R., Favory, J.J., Gruber, H., Bartelniewoehner, L., Bartels, S., Binkert, M., Funk, M., Weisshaar, B., and UIm, R. (2010). The Arabidopsis bZIP transcription factor HY5 regulates expression of the PFG1/MYB12 gene in response to light and ultraviolet-B radiation. Plant Cell Environ 33, 88-103.

Tavridou, E., Pireyre, M., and UIm, R. (2020). Degradation of the transcription factors PIF4 and PIF5 under UV-B promotes UVR8-mediated inhibition of hypocotyl growth in Arabidopsis. Plant J 101, 507-517.

Ulm, R., Baumann, A., Oravecz, A., Mate, Z., Adam, E., Oakeley, E.J., Schafer, E., and Nagy, F. (2004). Genome-wide analysis of gene expression reveals function of the bZIP transcription factor HY5 in the UV-B response of Arabidopsis. Proc Natl Acad Sci USA 101, 1397-1402.

Vojtek, A.B., and Hollenberg, S.M. (1995). Ras-Raf interaction: two-hybrid analysis. In Methods in Enzymology (Academic Press), pp. 331-342.

Wang, Z.-P., Xing, H.-L., Dong, L., Zhang, H.-Y., Han, C.-Y., Wang, X.-C., and Chen, Q.-J. (2015). Egg cellspecific promoter-controlled CRISPR/Cas9 efficiently generates homozygous mutants for multiple target genes in Arabidopsis in a single generation. Genome Biol 16, 144.

Wei, C.-Q., Chien, C.-W., Ai, L.-F., Zhao, J., Zhang, Z., Li, K.H., Burlingame, A.L., Sun, Y., and Wang, Z.-Y. (2016). The Arabidopsis B-box protein BZS1/BBX20 interacts with HY5 and mediates strigolactone regulation of photomorphogenesis. J Genet Genomics 43, 555-563.

Xu, D., Jiang, Y., Li, J., Lin, F., Holm, M., and Deng, X.W. (2016). BBX21, an Arabidopsis B-box protein, directly activates HY5 and is targeted by COP1 for $26 \mathrm{~S}$ proteasome-mediated degradation. Proc Natl Acad Sci USA 113, 7655-7660.

Yan, H., Marquardt, K., Indorf, M., Jutt, D., Kircher, S., Neuhaus, G., and Rodríguez-Franco, M. (2011). Nuclear localization and interaction with COP1 are required for STO/BBX24 function during photomorphogenesis. Plant Physiol 156, 1772-1782.

Yang, Y., Liang, T., Zhang, L., Shao, K., Gu, X., Shang, R., Shi, N., Li, X., Zhang, P., and Liu, H. (2018). UVR8 interacts with WRKY36 to regulate HY5 transcription and hypocotyl elongation in Arabidopsis. Nat Plants 4, 98-107.

Yin, R., Arongaus, A.B., Binkert, M., and UIm, R. (2015). Two distinct domains of the UVR8 photoreceptor interact with COP1 to initiate UV-B signaling in Arabidopsis. Plant Cell 27, 202213.

Yin, R., Skvortsova, M.Y., Loubery, S., and UIm, R. (2016). COP1 is required for UV-B-induced nuclear accumulation of the UVR8 photoreceptor. Proc Natl Acad Sci USA 113, E4415-E4422.

Yin, R., Messner, B., Faus-Kessler, T., Hoffmann, T., Schwab, W., Hajirezaei, M.-R., von Saint Paul, V., Heller, W., and Schäffner, A.R. (2012). Feedback inhibition of the general phenylpropanoid and flavonol biosynthetic pathways upon a compromised flavonol-3-O-glycosylation. J Exp Bot 63, 2465-2478. 
bioRxiv preprint doi: https://doi.org/10.1101/2021.10.14.464399; this version posted October 14,2021 . The copyright holder for this preprint

(which was not certified by peer review) is the author/funder, who has granted bioRxiv a license to display the preprint in perpetuity. It is made available under aCC-BY-NC-ND 4.0 International license.

721

722

723

724

725

726

Zhang, X., Huai, J., Shang, F., Xu, G., Tang, W., Jing, Y., and Lin, R. (2017). A PIF1/PIF3-HY5-BBX23 transcription factor cascade affects photomorphogenesis. Plant Physiol 174, 2487-2500.

Zoulias, N., Brown, J., Rowe, J., and Casson, S.A. (2020). HY5 is not integral to light mediated stomatal development in Arabidopsis. PLoS ONE 15, e0222480. 
bioRxiv preprint doi: https://doi.org/10.1101/2021.10.14.464399; this version posted October 14, 2021. The copyright holder for this preprint (which was not certified by peer review) is the author/funder, who has granted bioRxiv a license to display the preprint in perpetuity. It is made available under aCC-BY-NC-ND 4.0 International license.

A
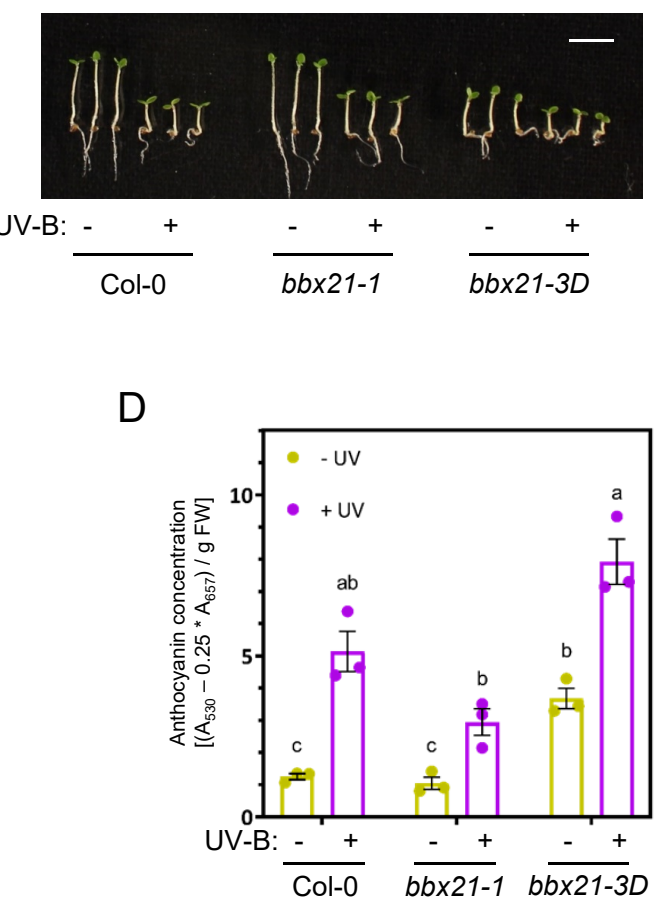

B

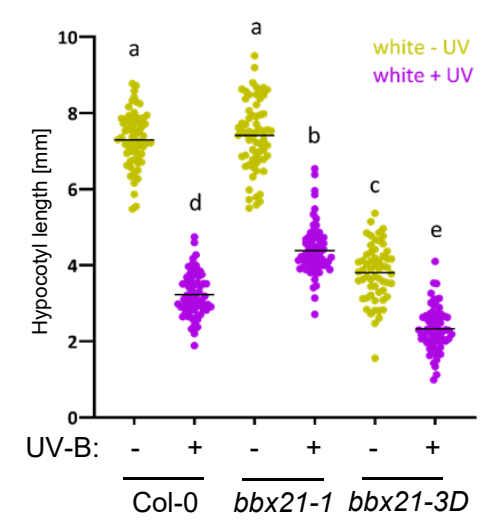

$\mathrm{C}$

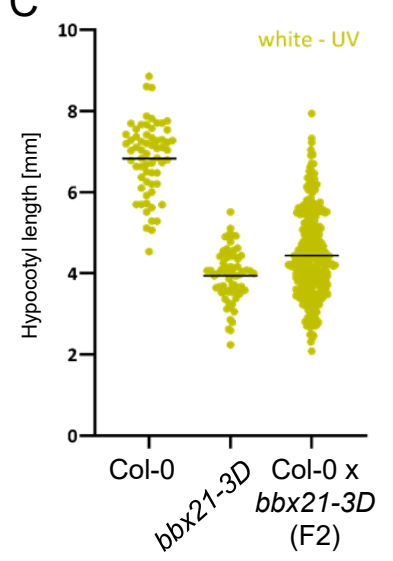

$E$

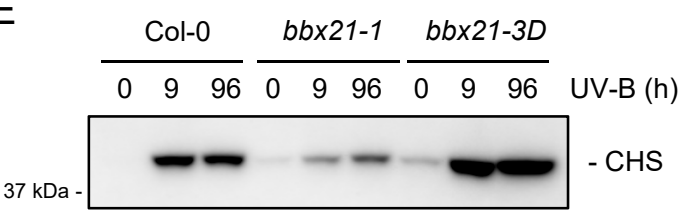

$50 \mathrm{kDa}-$

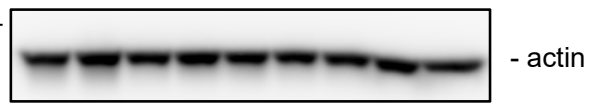

G

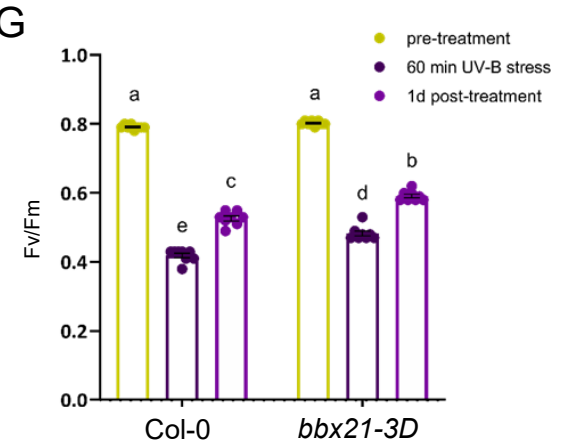

Figure $1 b b \times 21-3 D$ is a hypermorphic allele of BBX21. A, Representative images of wild type (Col-0) and bbx21-1 null and $b b \times 21-3 D$ gain-of-function mutant seedlings in white light supplemented with UV-B (+), or not (-). Scale bar indicates $5 \mathrm{~mm}$. B, Quantification of hypocotyl length of seedlings as shown in (A). C, Quantification of hypocotyl lengths of Col-0, bbx21-3D, and segregating F2 seedlings of a Col- $0 \times b b \times 21-3 D$ cross grown for $4 \mathrm{~d}$ in white light. $\mathrm{D}$, Anthocyanin concentrations in seedlings as shown in (A). E, Immunoblot analysis of CHS and actin (loading control) levels in Col-0, bbx21-1, and $b b x 21-3 D$ seedlings exposed to $9 \mathrm{~h}$ and $96 \mathrm{~h}$ of supplemental UV-B, or not (0). F, Immunoblot analysis of HY5 and actin (loading control) levels in Col-0, bbx21-1, bbx21-3D and hy5 seedlings exposed to $6 \mathrm{~h}$ and $96 \mathrm{~h}$ of supplemental UV-B, or not (0). G, Maximum efficiency of photosystem II (Fv/Fm) in wild-type (Col-0) and $b b \times 21-3 D$ seedlings grown for $7 \mathrm{~d}$ in white light ("pre-treatment"), exposed to 60 min of broadband UV-B ("60 min UV-B stress"), and left in white light for 1d of recovery ("1d post-treatment"). (B,C) Values of independent measurements and means as horizontal lines are shown; $n>60$. (D) Values of independent measurements, means, and SEM are shown $(n=3)$. (G) Values of independent measurements, means, and SEM are shown $(n=8)$. Shared letters indicate no statistically significant difference between the means $(P>0.05)$. 
bioRxiv preprint doi: https://doi.org/10.1101/2021.10.14.464399; this version posted October 14, 2021. The copyright holder for this preprint (which was not certified by peer review) is the author/funder, who has granted bioRxiv a license to display the preprint in perpetuity. It is made available under aCC-BY-NC-ND 4.0 International license.

A

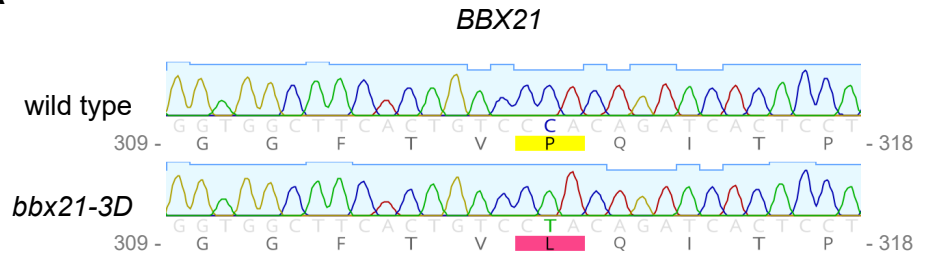

B

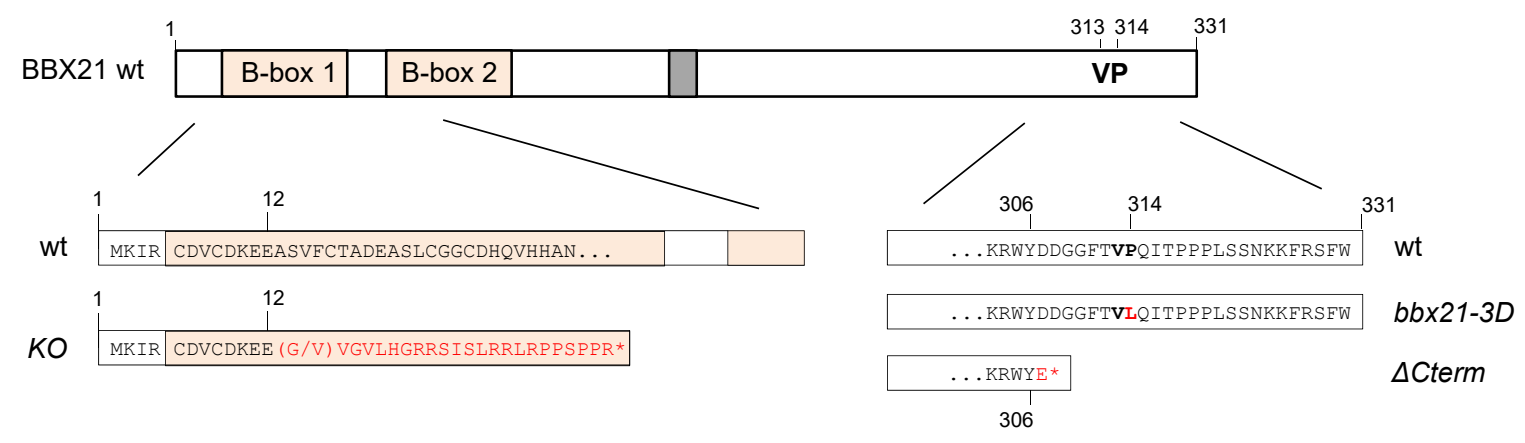

C

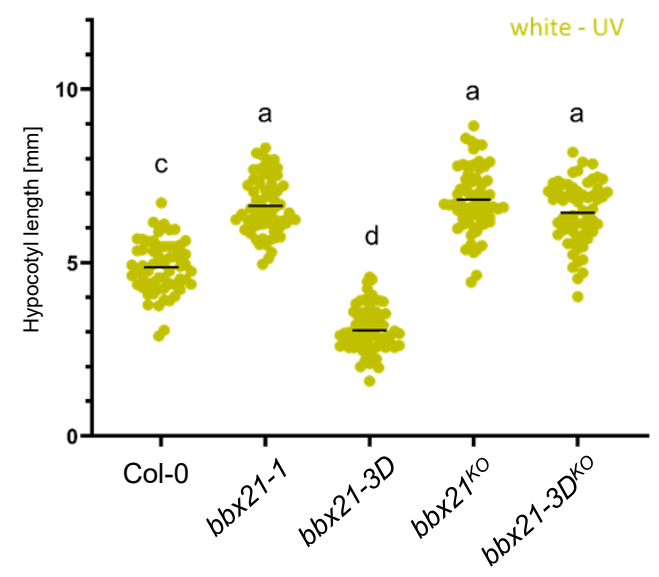

$\mathrm{D}$

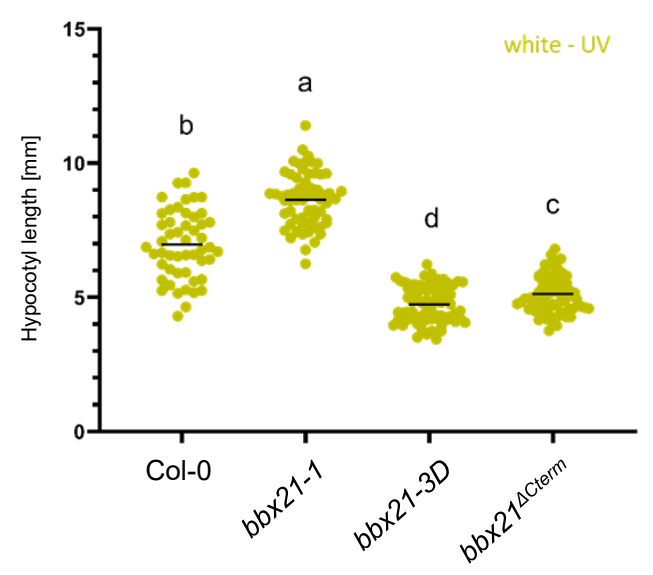

Supplemental Figure $\mathbf{S 1}$ The $b b \times 21-3 D$ phenotype is dependent on functional $B B X 21$, and can be recapitulated by deleting the C-terminal 25 amino acids that include the VP motif. A, Sequencing chromatograms depicting the C-to-T mutation in $b b \times 21-3 D$, generating the $\mathrm{BBX} 21^{\mathrm{P} 314 \mathrm{~L}}$ variant. $\mathrm{B}$, Schematic view of the $B B X 21$ coding sequence in wild type (wt), $b b \times 21-3 D$, and CRISPR/Cas9-mediated $b b \times 21$ mutant lines ( $K O$ and $\triangle C$ term). The positions of the two B-box domains, the transactivation domain (grey box), and VP motif are indicated. The altered amino acid residues generated due to the frameshift mutations are indicated in red, with * representing premature stop codons. KO, knock-out. C, Quantification of hypocotyl lengths of wild type (Col-0), bbx21-1, bbx21-3D, and bbx21 CRISPR/Cas9-mediated knockout lines in the Col-0 (bbx21KO) and bbx21-3D (bbx21$3 D^{K O}$ ) backgrounds. D, Quantification of hypocotyl lengths of Col-0, bbx21-1, bbx21-3D, and a C-terminally truncated CRISPR/Cas9-generated mutant line $\left(b b \times 21^{\Delta C t e r m}\right)$. (C,D) Seedlings were grown for $4 \mathrm{~d}$ in white light (values of independent measurements and means as horizontal lines are shown; $C, n>60 ; \mathrm{D}, n>50$ ). Shared letters indicate no statistically significant difference between the means $(P>0.05)$. 
A

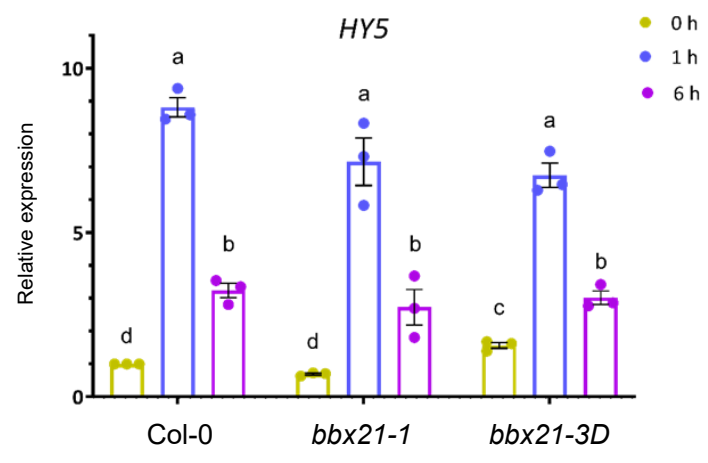

C

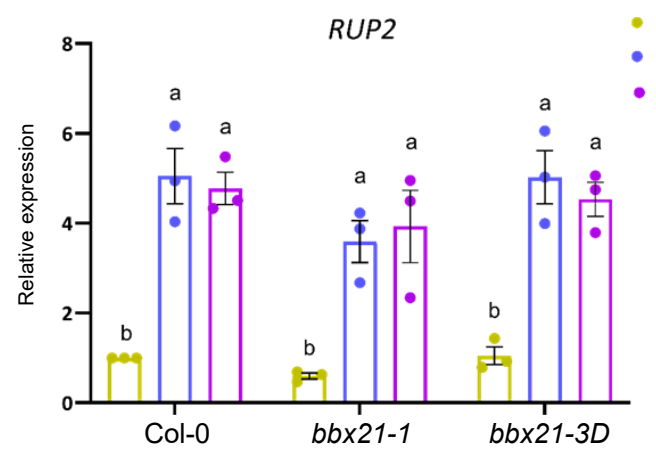

$\mathrm{E}$

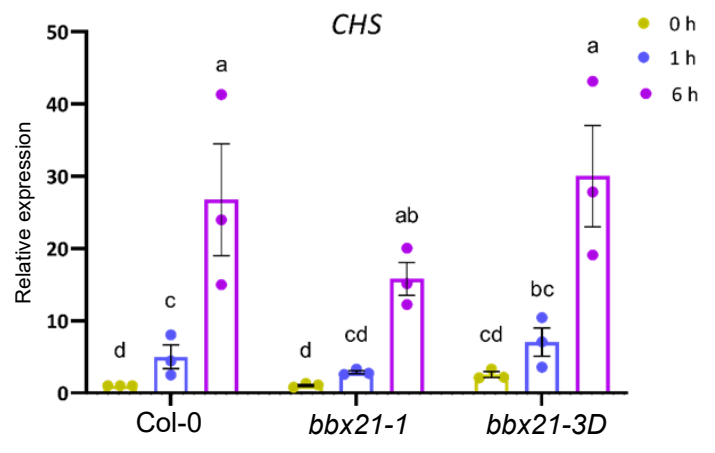

B

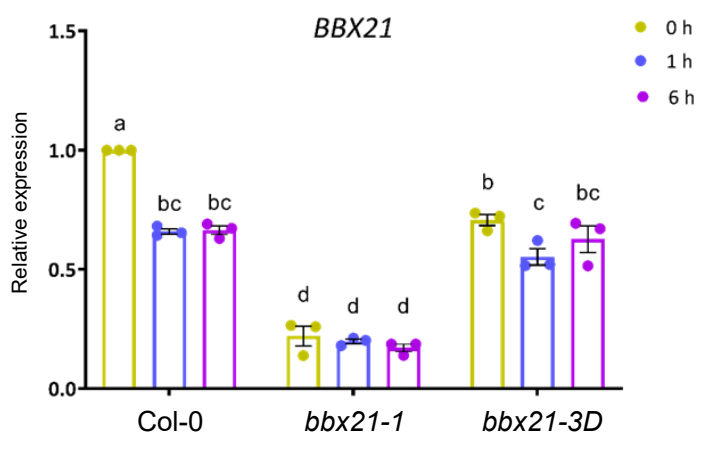

$\mathrm{D}$

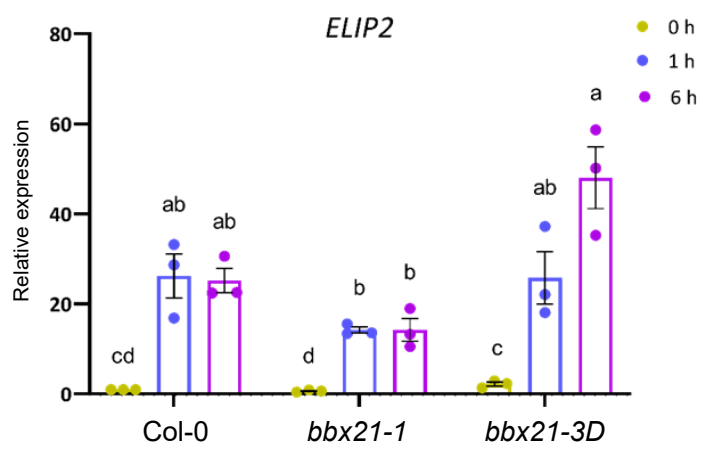

$\mathrm{F}$

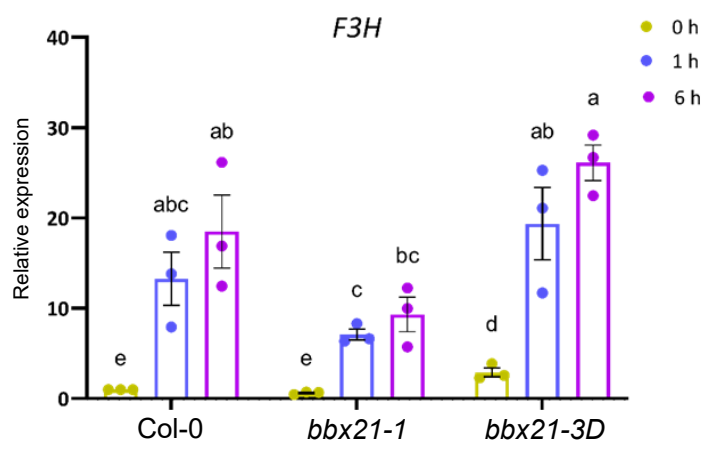

Supplemental Figure S2 BBX21 promotes the expression of some marker genes in response to UV-B. A-F, RT-qPCR analysis of $H Y 5$ (A), BBX21 (B), RUP2 (C), ELIP2 (D), CHS (E), and F3H (F) expression in 4-d-old wild-type (Col-0), bbx21-1, and bbx21$3 D$ seedlings grown in white light and exposed to $1 \mathrm{~h}$ and $6 \mathrm{~h}$ of supplemental UV-B, or not $(0 \mathrm{~h})$. Values of independent measurements, means, and SEM are shown $(n=3)$; shared letters indicate no statistically significant difference between the means $(P>0.05)$. 
bioRxiv preprint doi: https://doi.org/10.1101/2021.10.14.464399; this version posted October 14,2021 . The copyright holder for this preprint (which was not certified by peer review) is the author/funder, who has granted bioRxiv a license to display the preprint in perpetuity. It is made available under aCC-BY-NC-ND 4.0 International license.

A

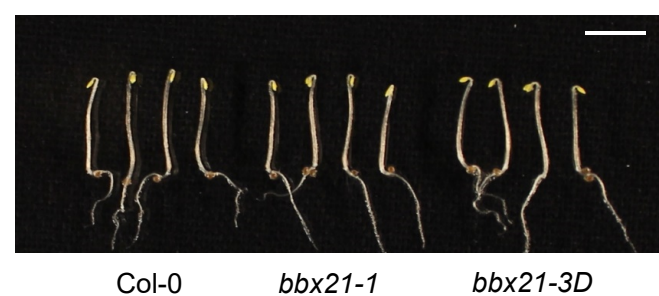

Col-0

$b b \times 21-1$

$b b \times 21-3 D$
B

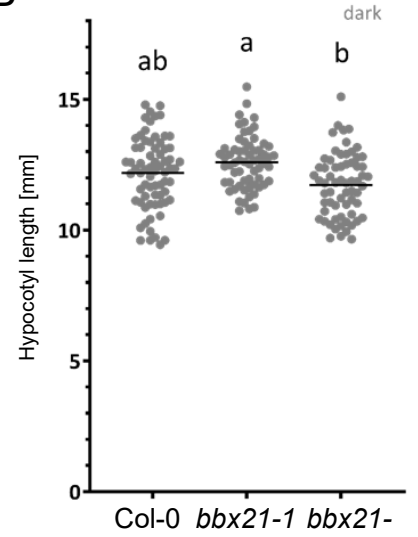

$3 D$

E
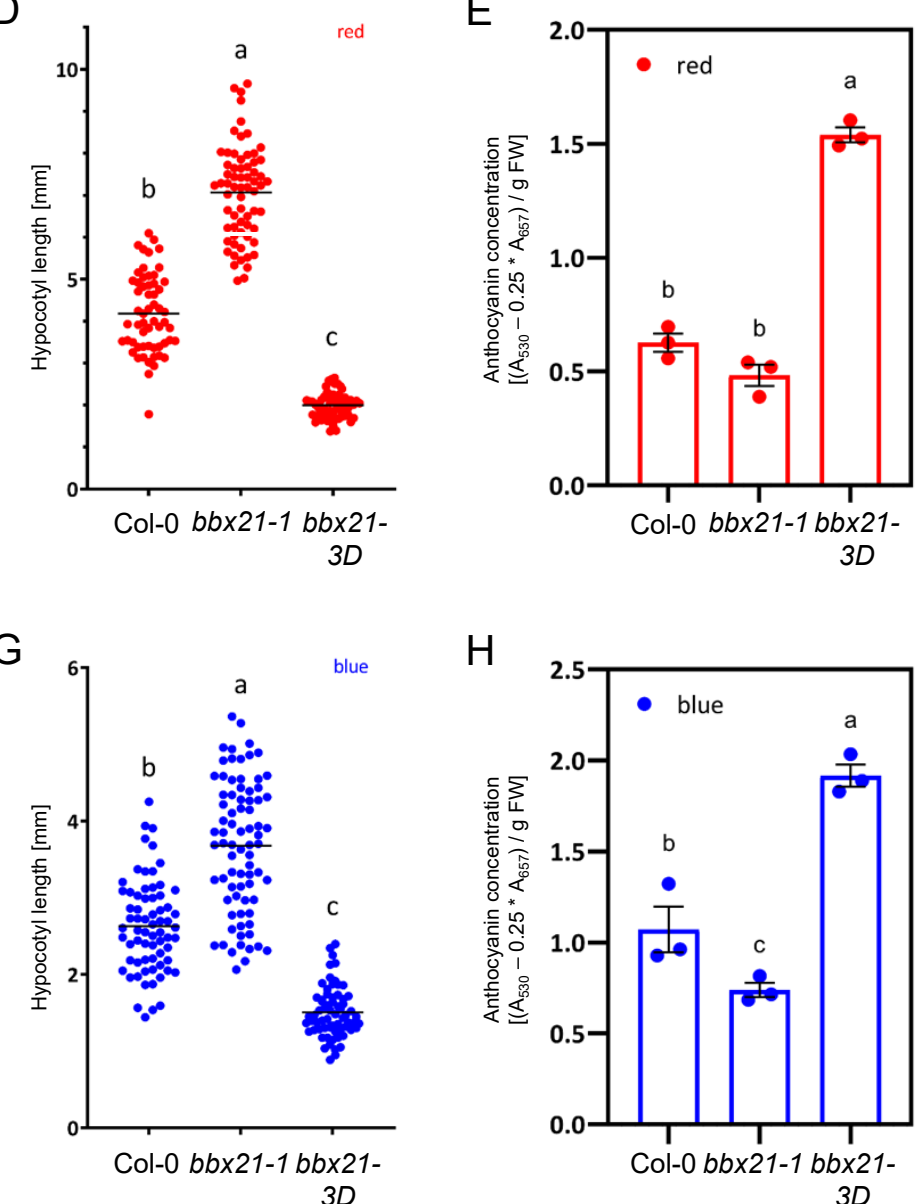

$\mathrm{H}$

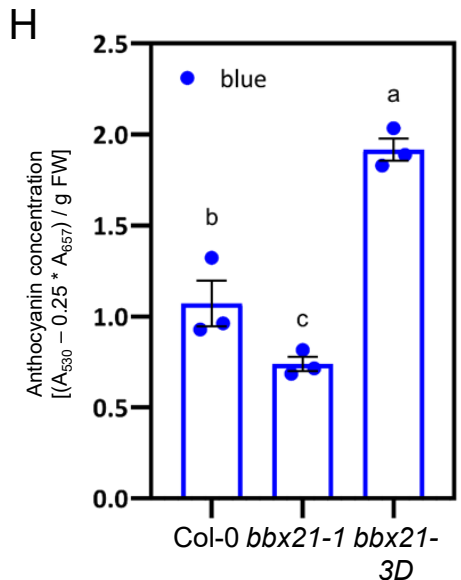

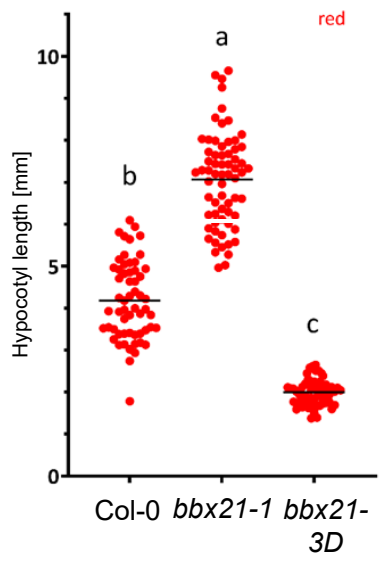

$\mathrm{F}$

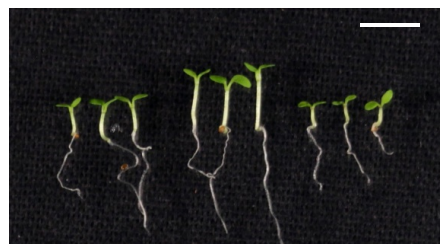

Col-0

$b b \times 21-1 \quad b b \times 21$

$3 D$

Supplemental Figure S3 bbx21-3D phenotype in darkness and monochromatic red and blue light. A-B, Representative images (A) and quantification of hypocotyl lengths (B) in wild type (Col-0) as well as bbx21-1 null and $b b x 21-3 D$ gain-of-function mutant seedlings grown in the dark. C-E, Representative images (C), quantification of hypocotyl lengths (D), and quantification of anthocyanin concentrations (E) in Col-0 as well as $b b \times 21-1$ null and $b b \times 21-3 D$ gain-of-function mutant seedlings grown under $150 \mu \mathrm{mol} \mathrm{m}^{-2} \mathrm{~s}^{-1}$ of red light $(n>60)$. $\mathrm{F}-\mathrm{H}$, Representative images (F), quantification of hypocotyl lengths $(G)$, and quantification of anthocyanin concentrations $(H)$ in Col-0 as well as $b b \times 21-1$ null and $b b \times 21-3 D$ gain-of-function mutant seedlings grown under $50 \mu \mathrm{mol} \mathrm{m}^{-2} \mathrm{~s}^{-1}$ of blue light. (A,C,F) Scale bar indicates 5 $\mathrm{mm}$. $(B, D, G)$ Values of independent measurements and means as horizontal lines are shown $(n>60)$. $(E, H)$ Values of independent measurements, means, and SEM are shown $(n=3)$. Shared letters indicate no statistically significant difference between the means $(\mathrm{P}>$ 0.05). 
bioRxiv preprint doi: https://doi.org/10.1101/2021.10.14.464399; this version posted October 14,2021 . The copyright holder for this preprint (which was not certified by peer review) is the author/funder, who has granted bioRxiv a license to display the preprint in perpetuity. It is made available under aCC-BY-NC-ND 4.0 International license.

A

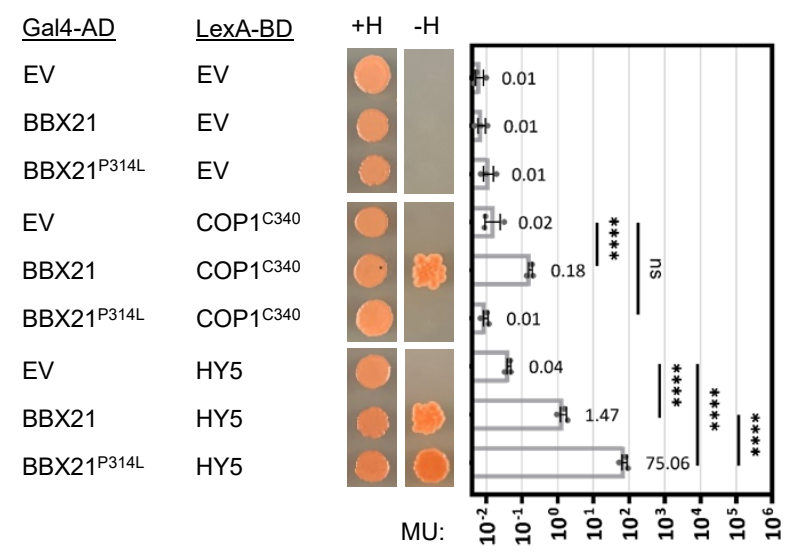

B

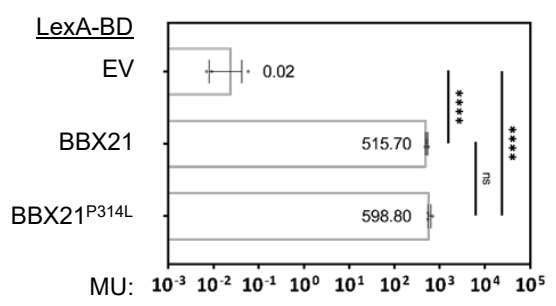

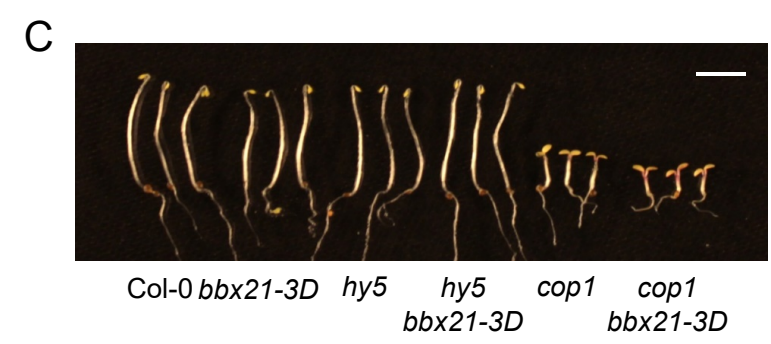

D

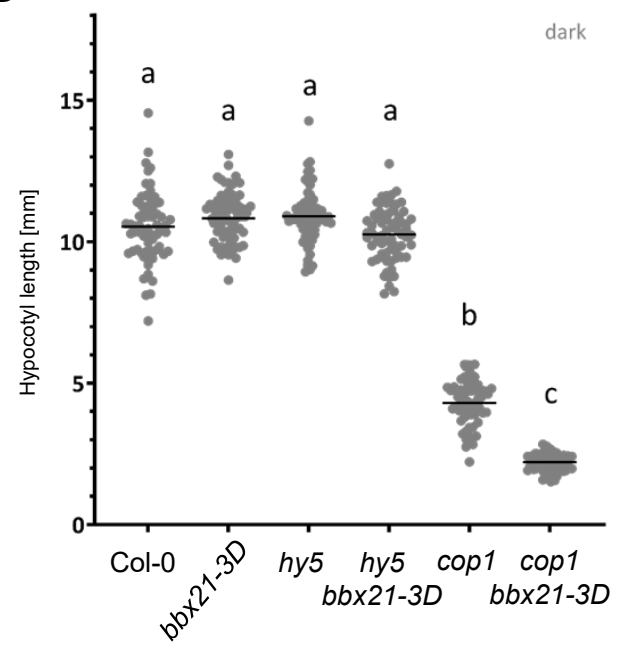

$\mathrm{E}$

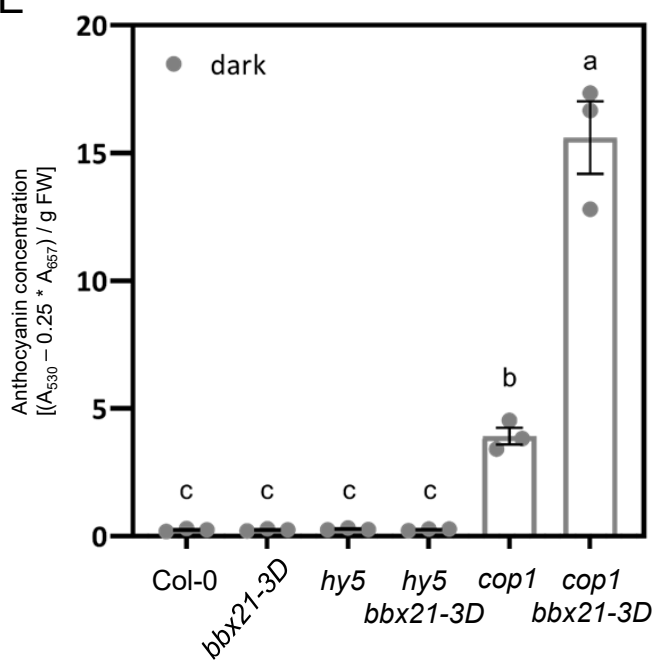

Figure 2 The BBX21 $314 \mathrm{~L}$ variant shows higher activity in a both COP1-dependent and -independent manner. A, Yeast two-hybrid analysis of the interactions of $\mathrm{BBX} 21$ and $\mathrm{BBX} 21^{\mathrm{P} 314 \mathrm{~L}}$ with $\mathrm{COP} 1^{\mathrm{C} 340}$ and HY5. Left: growth assay. Right: quantitative $\beta$-galactosidase assay. $\mathrm{AD}$, activation domain; $\mathrm{BD}$, DNA binding domain; $+\mathrm{H}$, +His medium (SD/-Trp/-Leu) as transformation control; - $\mathrm{H}$, selective -His medium (SD/-Trp/-Leu/-His); MU, Miller units; ns, non-significant $(P>0.05)$; ${ }^{* * *}, P<0.0001$. $B$, Analysis of the transactivation activity of BBX21 and BBX21 $1^{\text {314L }}$ fused to the LexA DNA binding domain using a quantitative $\beta$-galactosidase assay in yeast. BD, DNA binding domain; MU, Miller units; ns, non-significant $(P>0.05)$; ${ }^{* * \star *}, P<0.0001$. C-E, Representative images $(C$; scale bar indicates 5 mm), quantification of hypocotyl lengths ( $D$; values of independent measurements and means as horizontal lines are shown; $n>60)$, and quantification of anthocyanin concentrations (E; values of independent measurements, means, and SEM are shown; $n=3$ ) in wild-type (Col-0), bbx21-3D, hy5, hy5 bbx21-3D, cop1, and cop1 bbx21-3D seedlings grown in darkness. (D,E) Shared letters indicate no statistically significant difference between the means $(P>0.05)$. 
bioRxiv preprint doi: https://doi.org/10.1101/2021.10.14.464399; this version posted October 14,2021 . The copyright holder for this preprint (which was not certified by peer review) is the author/funder, who has granted bioRxiv a license to display the preprint in perpetuity. It is made available under aCC-BY-NC-ND 4.0 International license.

A
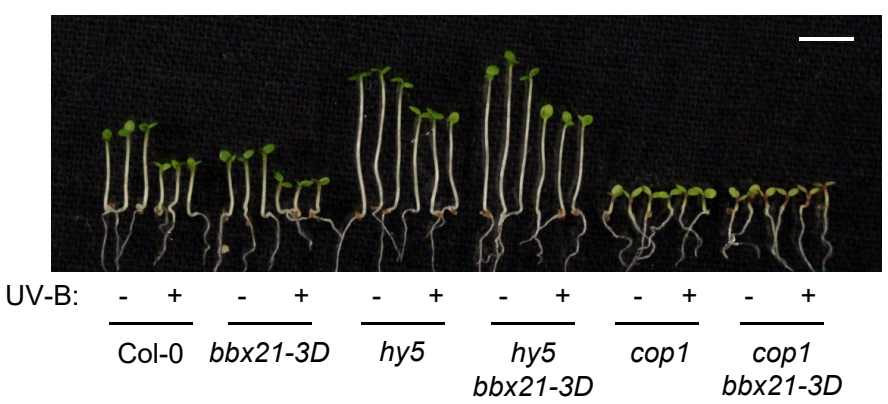

B

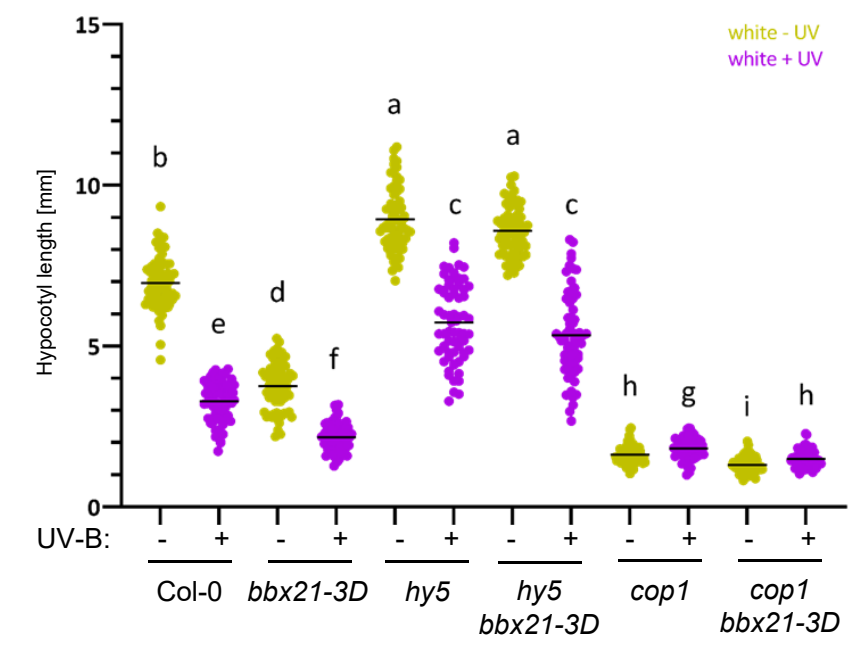

C

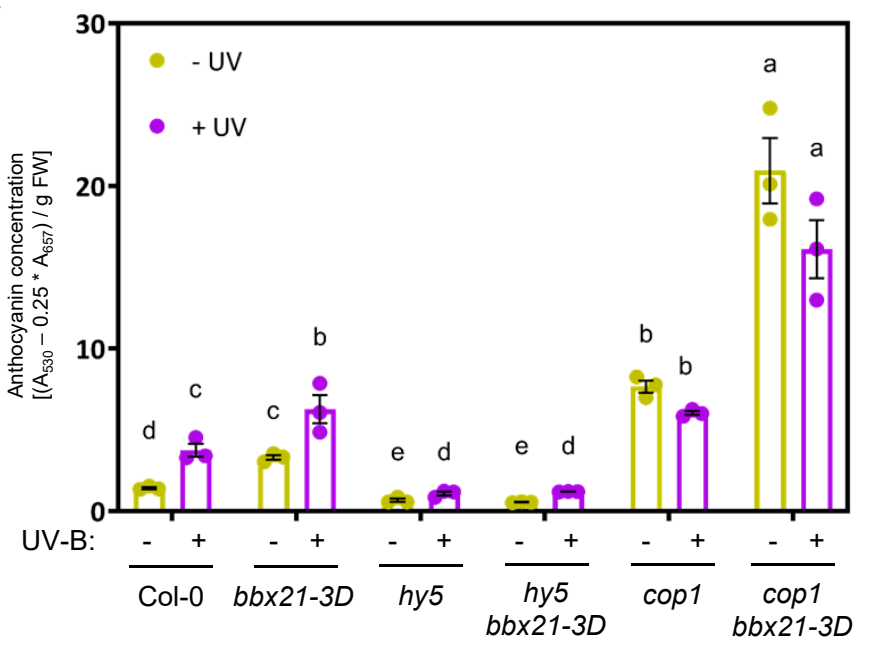

Figure 3 The $b b x 21-3 D$ phenotype requires functional HY5. A-C, Representative images (A; scale bar indicates $5 \mathrm{~mm}$ ), quantification of hypocotyl lengths (B; values of independent measurements and means as horizontal lines are shown; $n>60$ ), and quantification of anthocyanin concentrations (C; values of independent measurements, means, and SEM are shown; $n=3$ ) in wild-type (Col-0), bbx21-3D, hy5, hy5 bbx21-3D, cop1, and cop1 bbx21-3D seedlings grown in weak white light supplemented with UV-B $(+)$, or not $(-)$. (B,C) Shared letters indicate no statistically significant difference between the means $(P>0.05)$. 
bioRxiv preprint doi: https://doi.org/10.1101/2021.10.14.464399; this version posted October 14,2021 . The copyright holder for this preprint (which was not certified by peer review) is the author/funder, who has granted bioRxiv a license to display the preprint in perpetuity. It is made available under aCC-BY-NC-ND 4.0 International license.

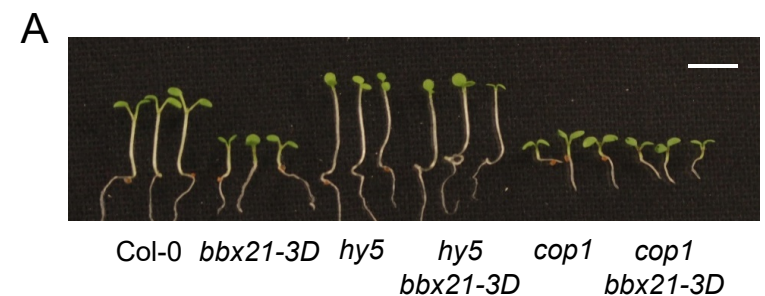

B

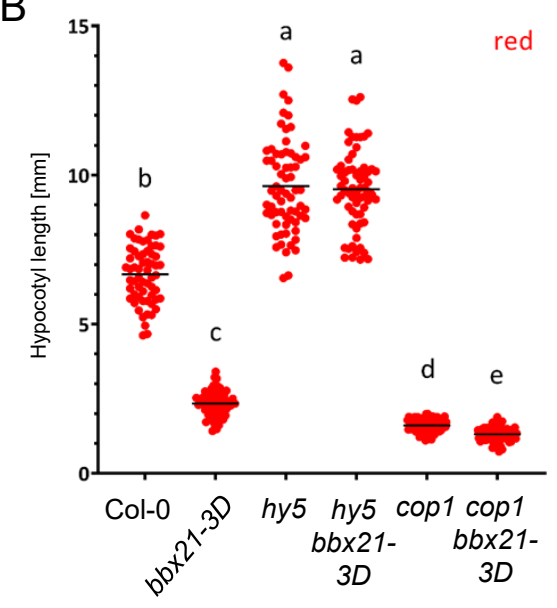

C

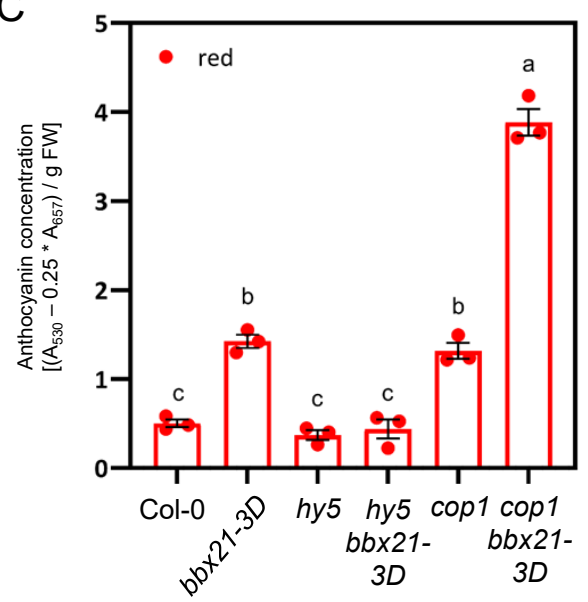

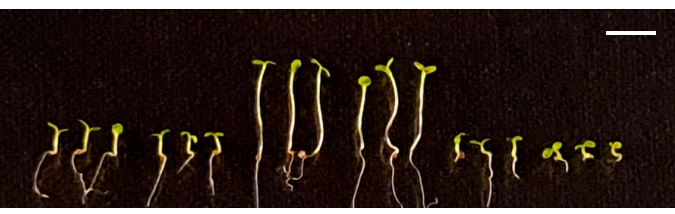

Col-0 bbx21-3D hy5 $\underset{b b \times 21-3 D}{\operatorname{cop} 1 \underset{b b \times 21-3 D}{\operatorname{cop} 1}}$

$\mathrm{E}$

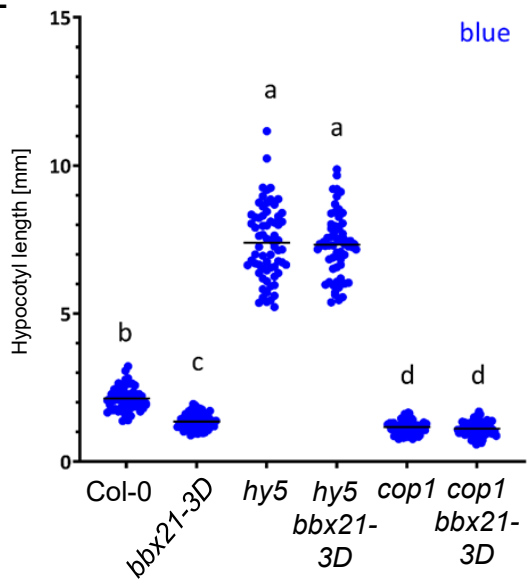

F

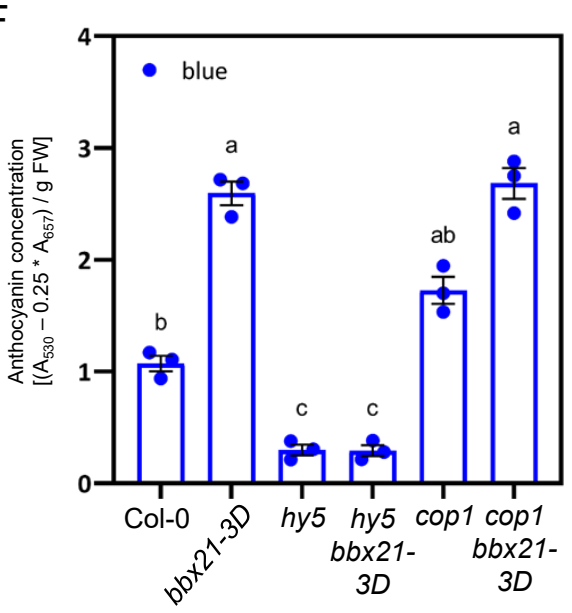

Supplemental Figure S4 Genetic relationship between bbx21-3D and cop1 and hy5 in monochromatic red and blue light conditions. A-F, Representative images (A,D; scale bars indicate $5 \mathrm{~mm}$ ), quantification of hypocotyl lengths (B,E; values of independent measurements and means as horizontal lines are shown; $n>60)$, and quantification of anthocyanin concentrations (C,F; values of independent measurements, means, and SEM are shown; $n=3$ ) in wild-type (Col-0), bbx21-3D, hy5, hy5 bbx21$3 D$, cop1, and cop1 bbx21-3D seedlings grown under $150 \mu \mathrm{mol} \mathrm{m}^{-2} \mathrm{~s}^{-1}$ of red light $(A-C)$ or $50 \mu \mathrm{mol} \mathrm{m}^{-2} \mathrm{~s}^{-1}$ of blue light (D-F). $(B, C, E, F)$ Shared letters indicate no statistically significant difference between the means $(P>0.05)$. 
bioRxiv preprint doi: https://doi.org/10.1101/2021.10.14.464399; this version posted October 14, 2021. The copyright holder for this preprint (which was not certified by peer review) is the author/funder, who has granted bioRxiv a license to display the preprint in perpetuity. It is made available under aCC-BY-NC-ND 4.0 International license.

A

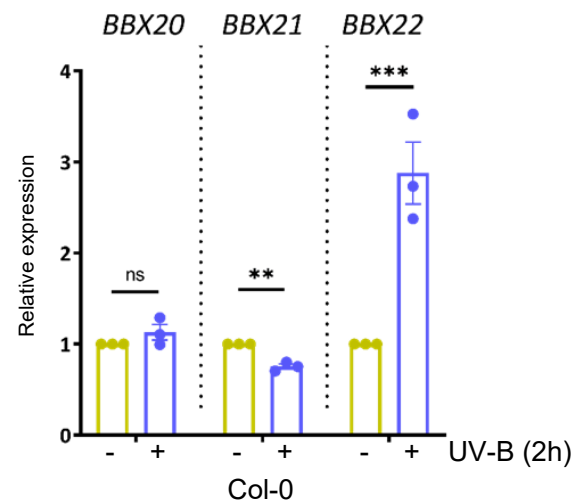

C
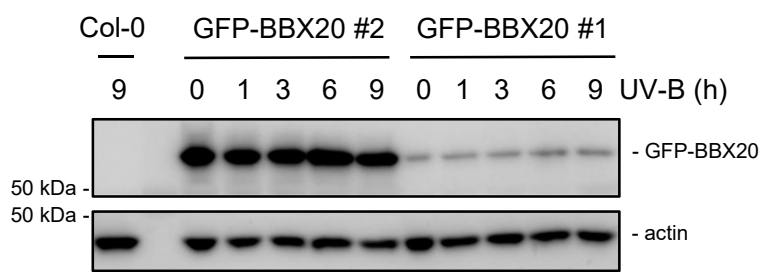

E
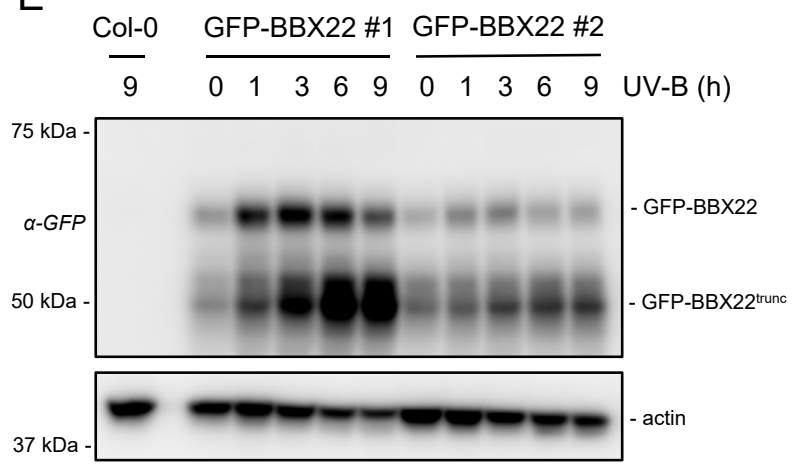

B

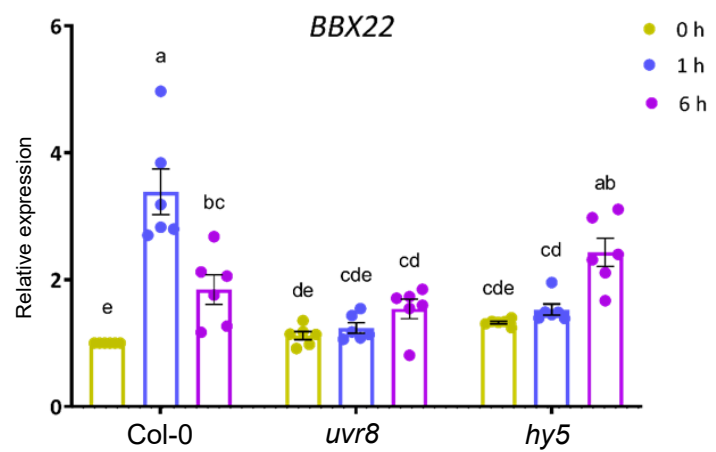

D
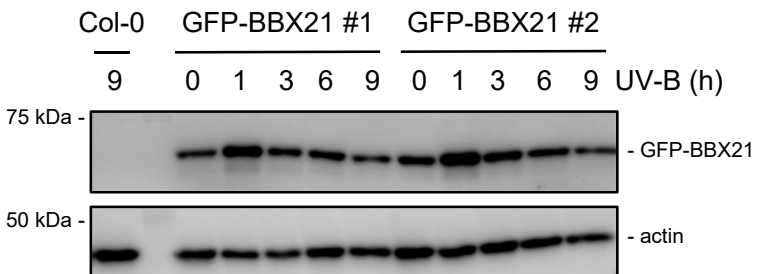

$\mathrm{F}$
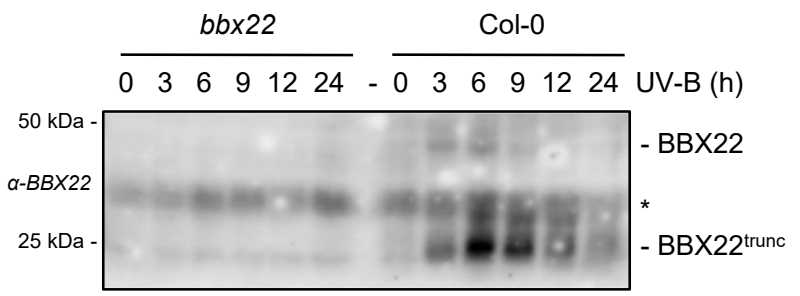

$37 \mathrm{kDa}$

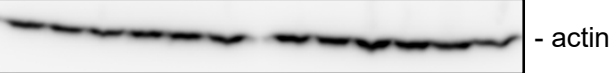

Figure 4 BBX21 and BBX22 are stabilized in response to UV-B. A, RT-qPCR analysis of $B B X 20, B B X 21$, and $B B X 22$ expression in 4-d-old wild-type (Col-0) seedlings grown in white light and exposed to $2 \mathrm{~h}$ of supplemental UV-B (blue bars), or not (dark yellow bars). B, RT-qPCR analysis of BBX22 expression in 4-d-old Col-0, uvr8-12 (uvr8), and hy5 seedlings grown in white light and exposed to $1 \mathrm{~h}$ and $6 \mathrm{~h}$ of supplemental UV-B, or not $(0 \mathrm{~h})$. C-E, Immunoblot analysis of GFP-BBX20 (C), GFP-BBX21 (D), GFP-BBX22 (E), and actin (loading control) levels in two independent lines of each Col-0/Pro ${ }_{35 s}:$ GFP-BBX20 (GFP-BBX20 \#1 and \#2), Col-0/Pro ${ }_{35 s}:$ GFP-BBX21 (GFP-BBX21 \#1 and \#2), and Col-0/Pro ${ }_{35 s}:$ GFP-BBX22 (GFP-BBX22 \#1 and \#2), with Col-0 as negative control. 4-d-old seedlings were treated for 0-9 $\mathrm{h}$ with supplemental UV-B, as indicated. F, Immunoblot analysis of endogenous BBX22 and actin (loading control) levels in bbx22 and Col-0 seedlings treated for 0-24 h with supplemental UV-B, as indicated. * indicates nonspecific cross-reacting bands. (A,B) Values of independent measurements, means, and SEM are shown (A, $n=3$; $\mathrm{B}, n=6$ ). (A) ns, non-significant $(\mathrm{P}>0.05) ;{ }^{* *}, \mathrm{P}<0.01$; ${ }^{* * *}, \mathrm{P}<0.001$. (B) Shared letters indicate no statistically significant difference between the means $(P>0.05)$. 
bioRxiv preprint doi: https://doi.org/10.1101/2021.10.14.464399; this version posted October 14, 2021. The copyright holder for this preprint (which was not certified by peer review) is the author/funder, who has granted bioRxiv a license to display the preprint in perpetuity. It is made available under aCC-BY-NC-ND 4.0 International license.
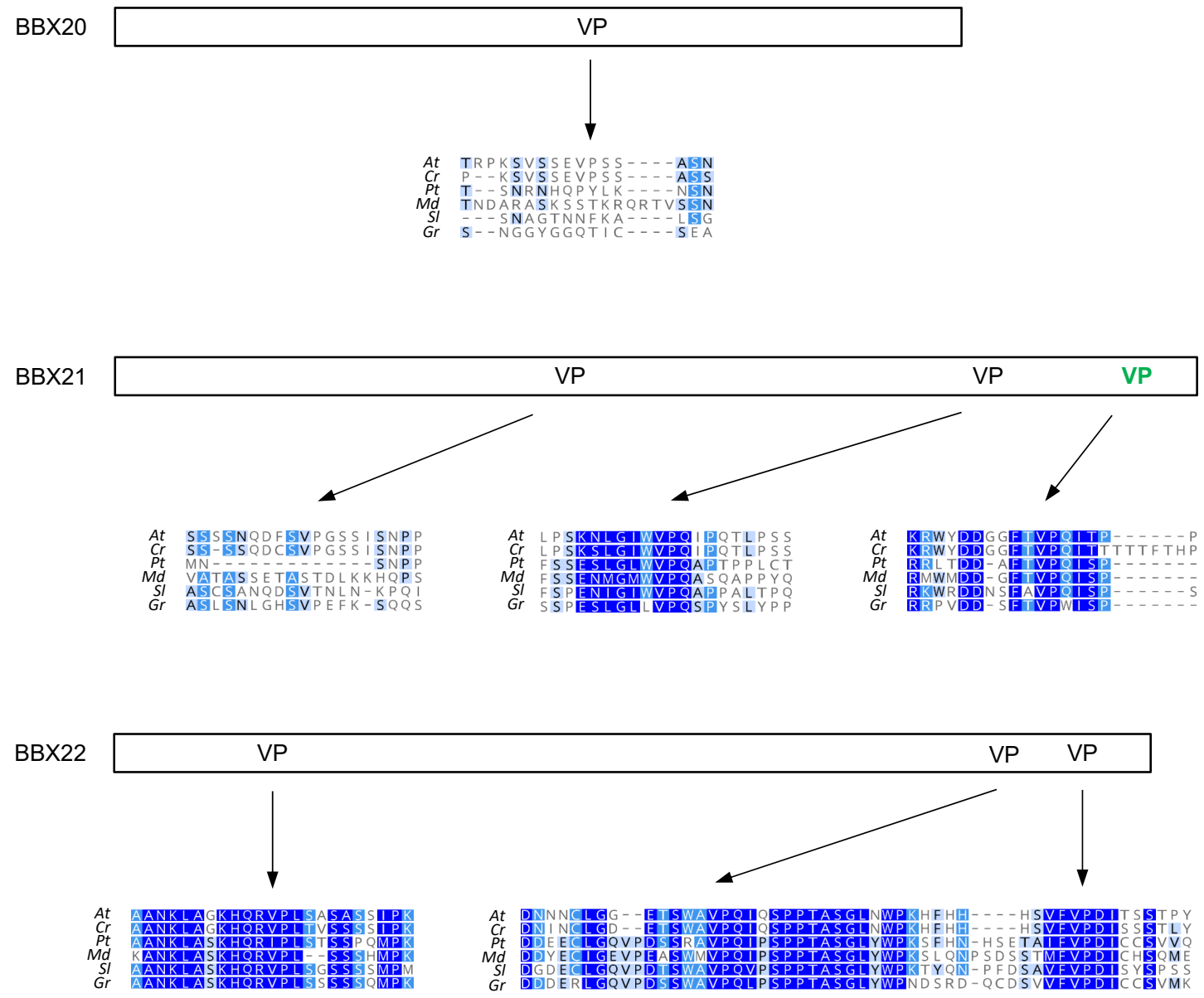

Supplemental Figure S5 Conservation of putative VP motifs in BBX20, BBX21, and BBX22. Schematic representation of the positions of all VP amino acid pairs in Arabidopsis BBX20, BBX21, and BBX22. In green, reported VP motif in BBX21 (Bursch et al., 2020). For each VP pair, an alignment of homologs, as determined by reciprocal BLASTs on the Phytozome resource (phytozome.jgi.doe.gov) is shown with conservation indicated in blue. Species analysed: At, Arabidopsis thaliana; $\mathrm{Cr}$, Capsella rubella; Pt, Populus trichocarpa; Md, Malus domestica; SI, Solanum lycopersicum, Gr, Gossypium raimondii. 
bioRxiv preprint doi: https://doi.org/10.1101/2021.10.14.464399; this version posted October 14, 2021. The copyright holder for this preprint (which was not certified by peer review) is the author/funder, who has granted bioRxiv a license to display the preprint in perpetuity. It is made available under aCC-BY-NC-ND 4.0 International license.

A

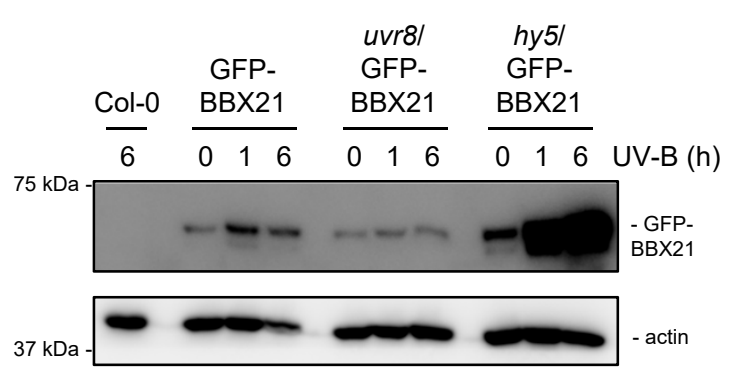

B

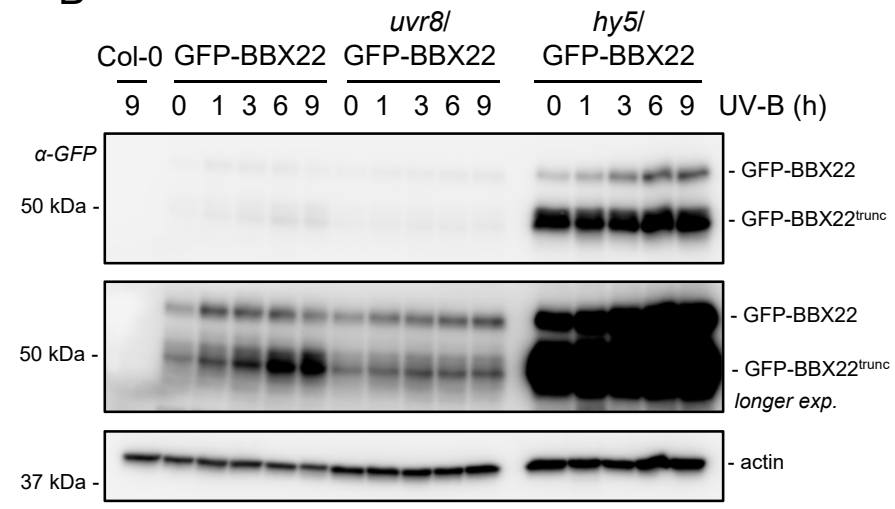

C

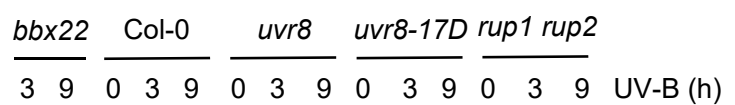

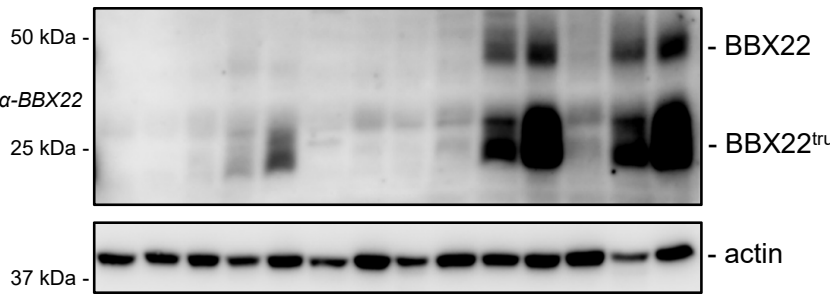

$\mathrm{E}$

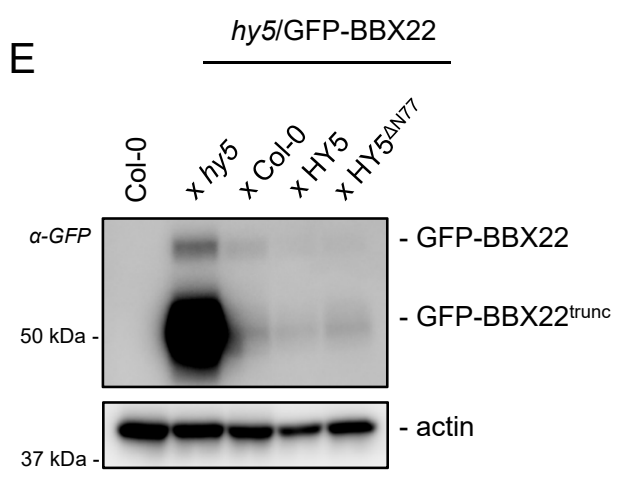

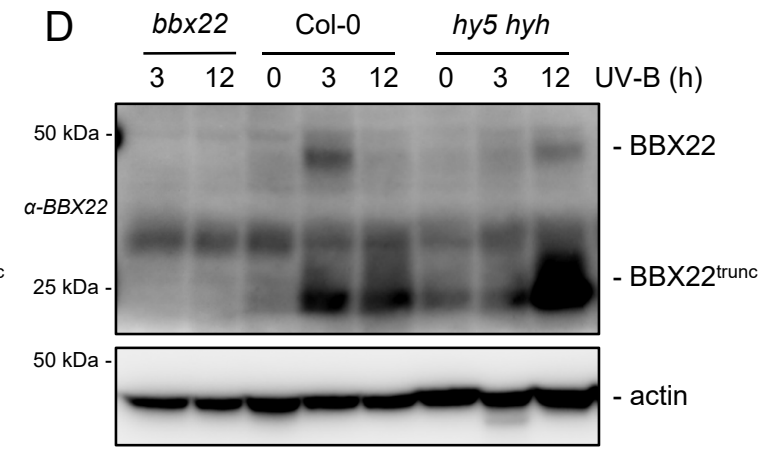

F

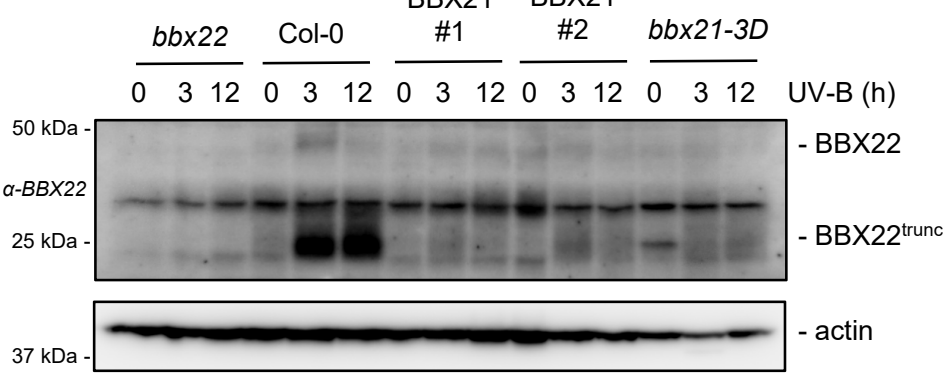

Figure 5 BBX21 and BBX22 accumulation under UV-B is UVR8-dependent and negatively regulated by HY5 activity. A,

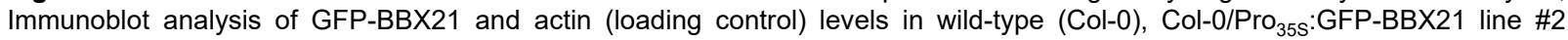
(GFP-BBX21), uvr8-12/Pro ${ }_{35 s}:$ GFP-BBX21 line \#2 (uvr8/GFP-BBX21), and hy5-215/Pro ${ }_{55 s}:$ GFP-BBX21 line \#2 (hy5/GFPBBX21) seedlings grown for $4 \mathrm{~d}$ and treated for $0-6 \mathrm{~h}$ with supplemental UV-B, as indicated. B, Immunoblot analysis of GFPBBX22 and actin (loading control) levels in Col-0, Col-0/Pro ${ }_{35 s}:$ GFP-BBX22 line \#1 (GFP-BBX22), uvr8-12/Pro ${ }_{35 s}:$ GFP-BBX22 line \#1 (uvr8/GFP-BBX22), and hy5-215/Pro ${ }_{35 s}$ :GFP-BBX22 line \#1 (hy5/GFP-BBX22) seedlings grown for $4 \mathrm{~d}$ and treated for $0-$ $9 \mathrm{~h}$ with supplemental UV-B, as indicated. C, Immunoblot analysis of endogenous BBX22 and actin (loading control) levels in bbx22, Col-0, uvr8-12 (uvr8), uvr8-17D, and rup1 rup2 seedlings treated for 0-9 h with supplemental UV-B, as indicated. D, Immunoblot analysis of endogenous BBX22 and actin (loading control) levels in bbx22, Col-0, and hy5 hyh seedlings treated for 0-12 h with supplemental UV-B, as indicated. E, Immunoblot analysis of GFP-BBX22 and actin (loading control) levels in Col-0, and $\mathrm{F} 1$ progeny from crosses of hy5/GFP-BBX22 with hy5, Col-0, hy5-215/Pro ${ }_{355}: \mathrm{HY} 5$ line \#15 (HY5), and hy5215/Pro ${ }_{355}: \mathrm{HY}^{\Delta \mathrm{N} 77}$ line \#27 (HY5 ${ }^{\Delta \mathrm{N} 77}$ ), grown for $4 \mathrm{~d}$ in white light. $\mathrm{F}$, Immunoblot analysis of endogenous BBX22 and actin (loading control) levels in bbx22, Col-0, Col-0/Pro ${ }_{35 s}:$ GFP-BBX21 \#1 (GFP-BBX21 \#1), Col-0/Pro 35 :GFP-BBX21 \#2 (GFP$\mathrm{BBX} 21 \mathrm{\# 2}$ ), and $b b \times 21-3 D$ seedlings treated for $0-12 \mathrm{~h}$ with supplemental UV-B, as indicated. 
bioRxiv preprint doi: https://doi.org/10.1101/2021.10.14.464399; this version posted October 14, 2021. The copyright holder for this preprint (which was not certified by peer review) is the author/funder, who has granted bioRxiv a license to display the preprint in perpetuity. It is made available under aCC-BY-NC-ND 4.0 International license.

A

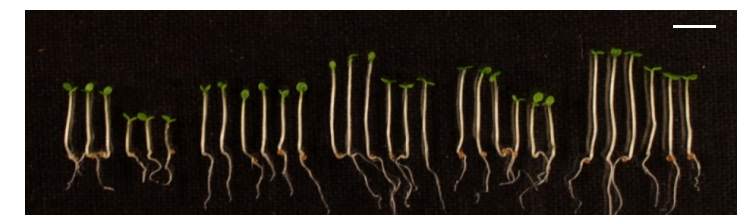

UV-B: $\frac{-}{\text { Col-0 }} \frac{-+}{\text { uvr8 }} \frac{-+}{\text { hy5 }} \quad \frac{-+}{b b \times 20} \frac{-+}{\text { hy5 }}$

$b b \times 21 \quad b b \times 20$

$b b \times 22 \quad b b \times 21$

$b b \times 22$

\section{C}

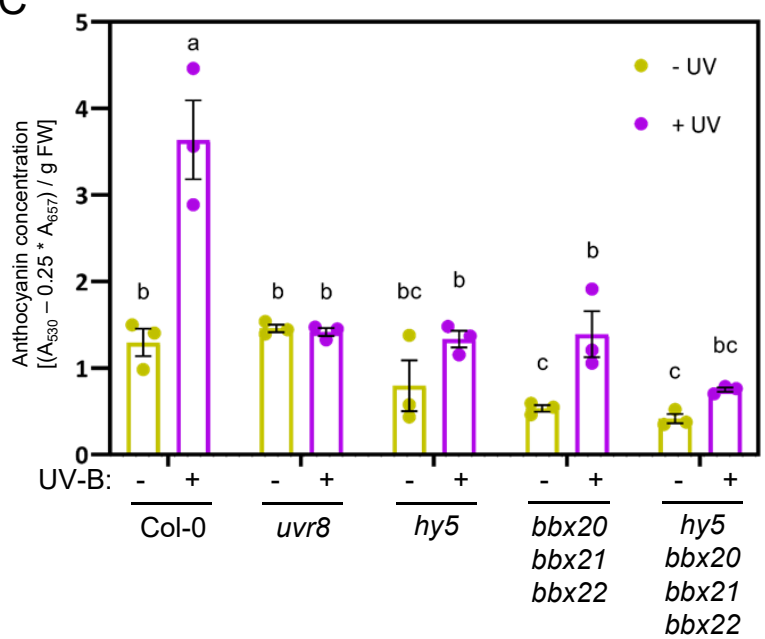

E
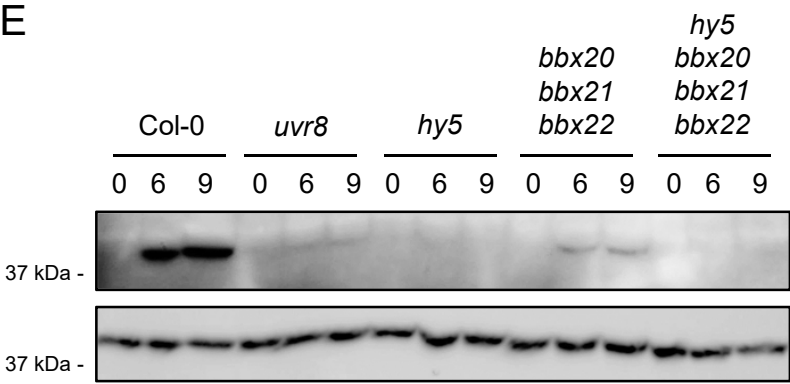

UV-B (h)

$-\mathrm{CHS}$

actin

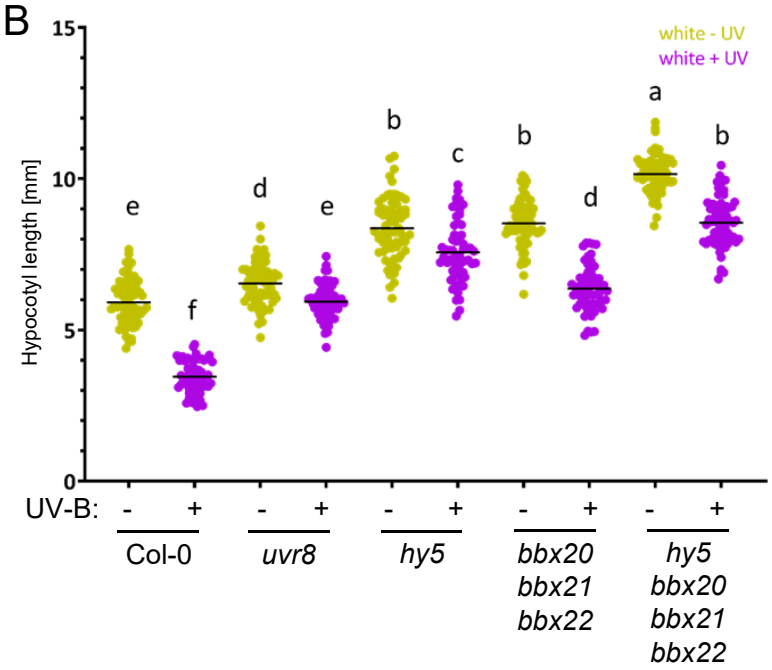

D

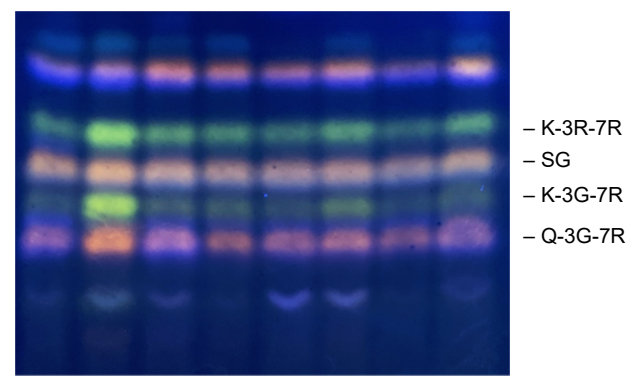

UV-B:

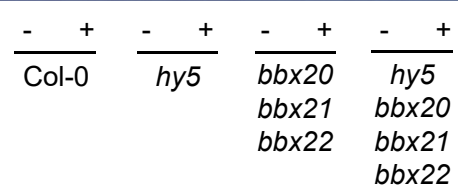

$\mathrm{F}$

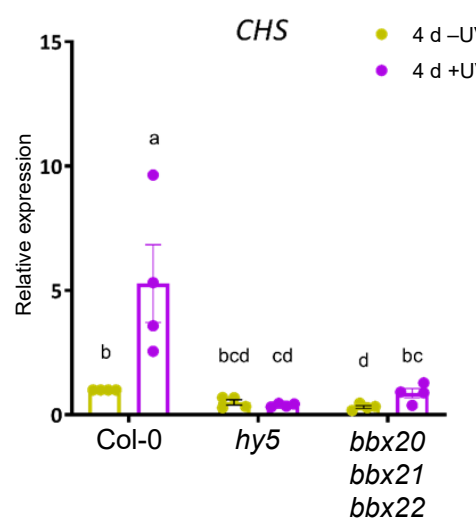

Figure 6 BBX proteins are necessary for hypocotyl growth inhibition and pigment accumulation under UV-B. A, Representative images of wild-type (Col-0), uvr8-12 (uvr8), hy5-215 (hy5), bbx20 bbx21 bbx22, and hy5 bbx20 bbx21 bbx22 mutant seedlings in white light supplemented (+) or not (-) with UV-B. Scale bar indicates $5 \mathrm{~mm}$. B, Quantification of hypocotyl length of seedlings as shown in (A). C, Anthocyanin concentrations in seedlings as shown in (A). D, HPTLC analysis of the flavonol glycoside levels in seedlings as described in (A) grown in white light or white light supplemented with UV-B. K-3R-7R, kaempferol-3-O-rhamnoside-7O-rhamnoside; SG, sinapoyl glucose; K-3G-7R, kaempferol-3-O-glucoside-7-O-rhamnoside; Q-3G-7R, quercetin-3-O-glucoside-7O-rhamnoside. E, Immunoblot analysis of CHS and actin (loading control) levels in seedlings as described in (A) that were exposed for $0-9 \mathrm{~h}$ to supplemental UV-B, as indicated. F, RT-qPCR analysis of CHS expression in Col-0, hy5, and bbx20 bbx21 bbx22 seedlings grown for $4 \mathrm{~d}$ in white light or white light supplemented with UV-B. (B) Values of independent measurements and means as horizontal lines are shown $(n>60)$. (C,F) Values of independent measurements, means, and SEM are shown $(\mathrm{C}, n=3 ; \mathrm{F}, n=$ 4). (B,C,F) Shared letters indicate no statistically significant difference between the means $(P>0.05)$. 
bioRxiv preprint doi: https://doi.org/10.1101/2021.10.14.464399; this version posted October 14, 2021. The copyright holder for this preprint (which was not certified by peer review) is the author/funder, who has granted bioRxiv a license to display the preprint in perpetuity. It is made available under aCC-BY-NC-ND 4.0 International license.

A

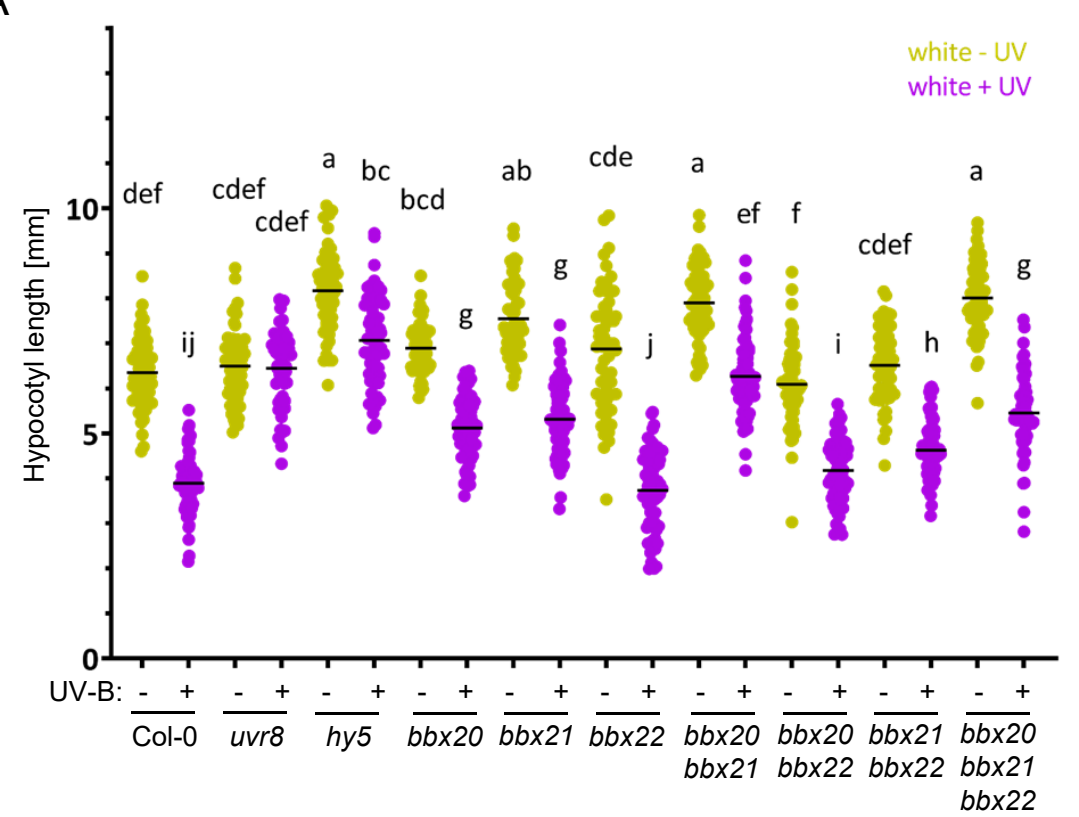

B
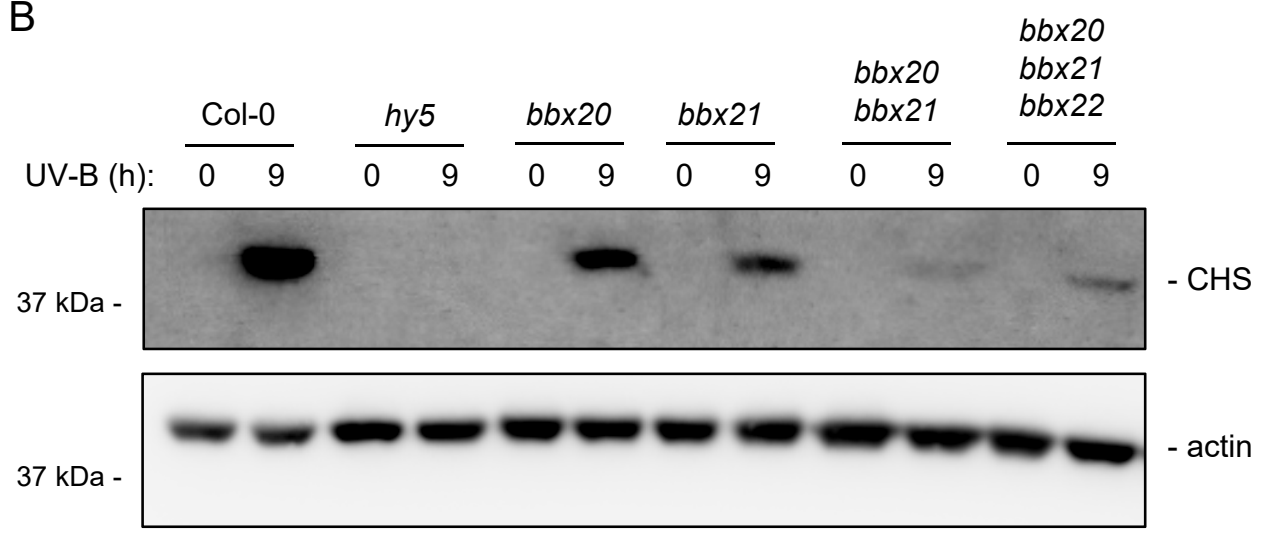

Supplemental Figure S6 Genetic redundancy between BBX20, BBX21, and BBX22. A, Quantification of hypocotyl lengths of seedlings of wild-type (Col-0), uvr8-12 (uvr8), hy5-215 (hy5), and single and combinatorial mutants of bbx20, bbx21, and bbx22. Values of independent measurements and means as horizontal lines are shown $(n>60)$. Shared letters indicate no statistically significant difference between the means ( $\mathrm{P}>0.05)$. B, Immunoblot analysis of CHS and actin (loading control) levels in Col-0, hy5, and single and combinatorial mutants of $b b \times 20, b b \times 21$, and $b b \times 22$. Seedlings were grown for $4 \mathrm{~d}$ and treated for $9 \mathrm{~h}$ with supplemental UV-B, or not (0). 
bioRxiv preprint doi: https://doi.org/10.1101/2021.10.14.464399; this version posted October 14, 2021. The copyright holder for this preprint (which was not certified by peer review) is the author/funder, who has granted bioRxiv a license to display the preprint in perpetuity. It is made available under aCC-BY-NC-ND 4.0 International license.

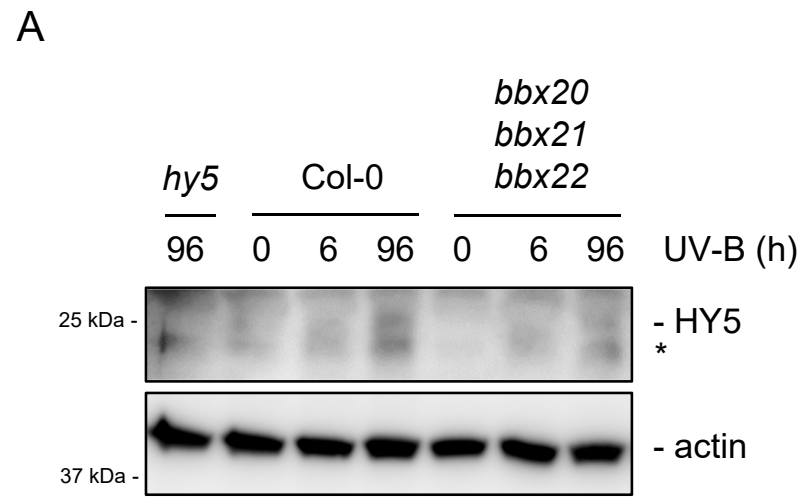

B

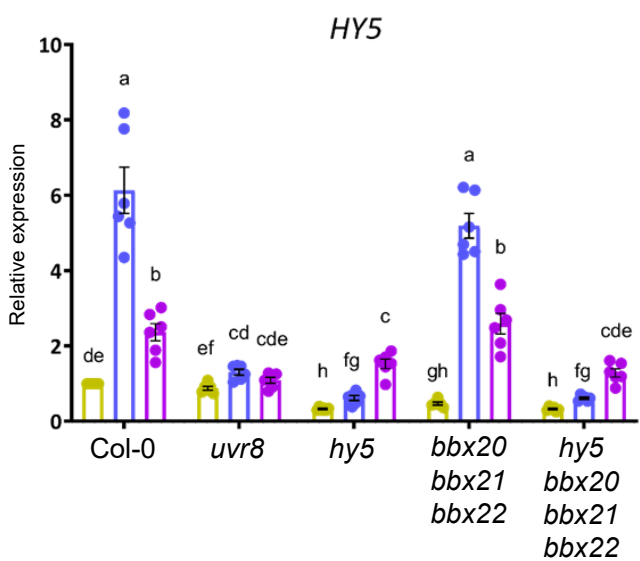

$\mathrm{D}$

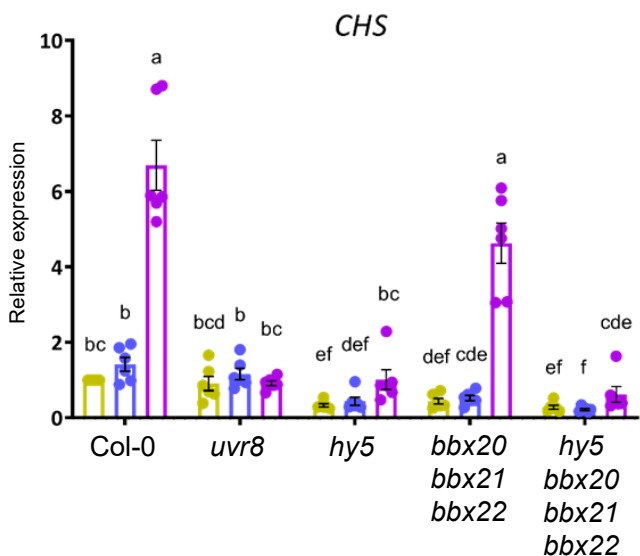

C

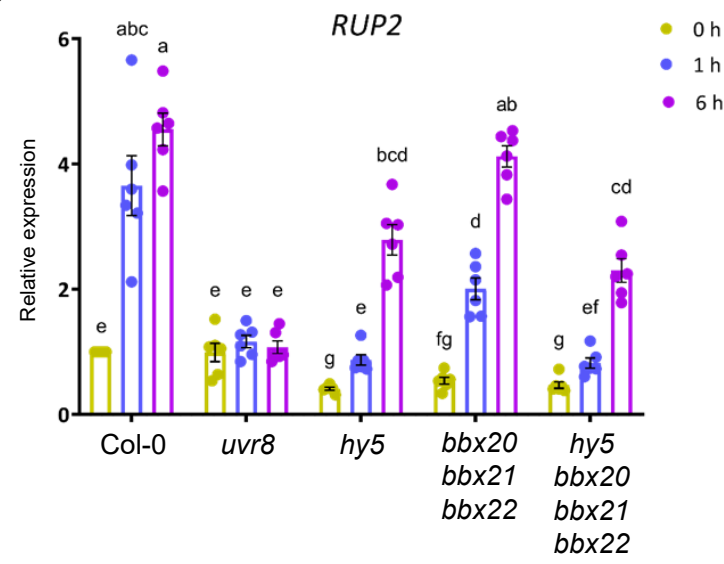

E

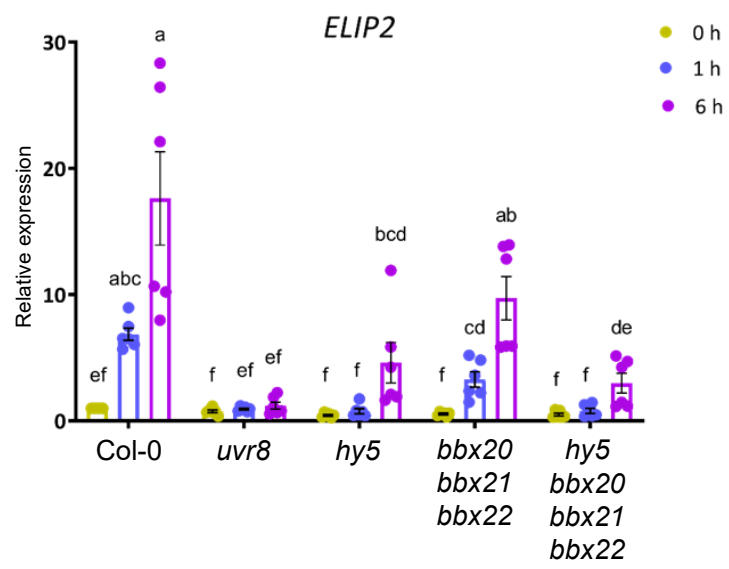

Figure 7 BBX proteins play a minor role in the short-term induction of UV-B marker genes. A, Immunoblot analysis of HY5 and actin (loading control) levels in wild-type (Col-0), hy5, and bbx20 bbx21 bbx22 seedlings exposed to $6 \mathrm{~h}$ and $96 \mathrm{~h}$ of supplemental UV-B, or not (0). B-E, RT-qPCR analysis of HY5 (B), RUP2 (C), CHS (D), and ELIP2 (E) expression in 4-d-old Col0 , uvr8, hy5, bbx20 bbx21 bbx22, and hy5 bbx20 bbx21 bbx22 seedlings grown in white light and exposed to $1 \mathrm{~h}$ and $6 \mathrm{~h}$ of supplemental UV-B, or not $(0 \mathrm{~h})$. Values of independent measurements, means, and SEM are shown $(n=6)$; shared letters indicate no statistically significant difference between the means $(P>0.05)$. 
bioRxiv preprint doi: https://doi.org/10.1101/2021.10.14.464399; this version posted October 14, 2021. The copyright holder for this preprint (which was not certified by peer review) is the author/funder, who has granted bioRxiv a license to display the preprint in perpetuity. It is made available under aCC-BY-NC-ND 4.0 International license.

A

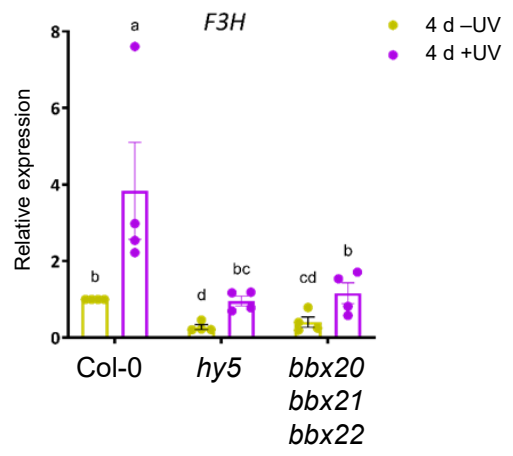

B

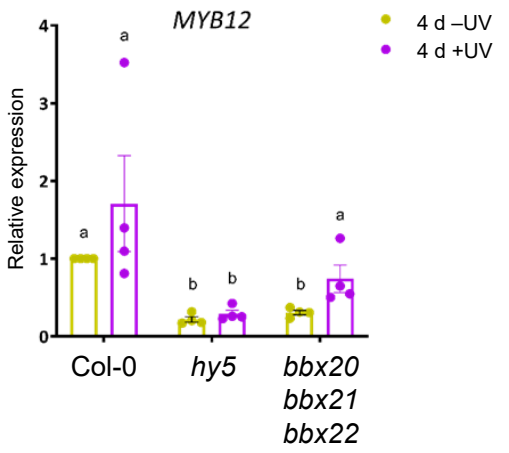

C

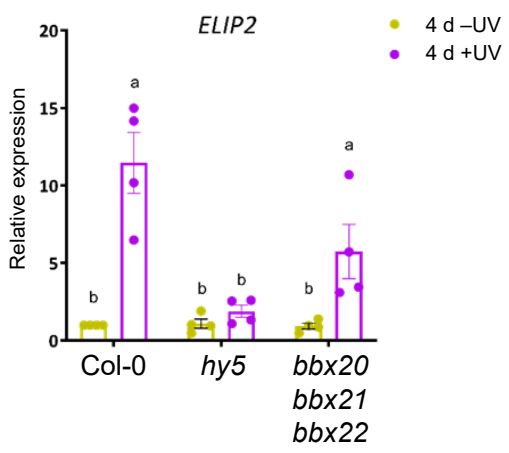

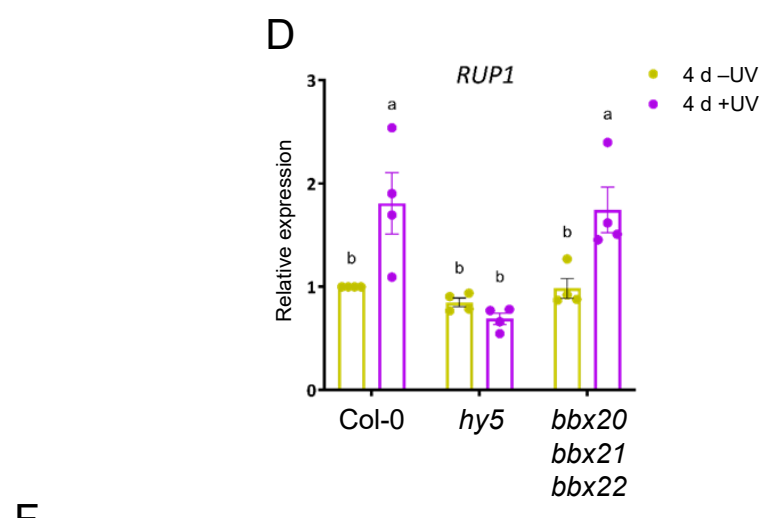

$E$

$\mathrm{F}$
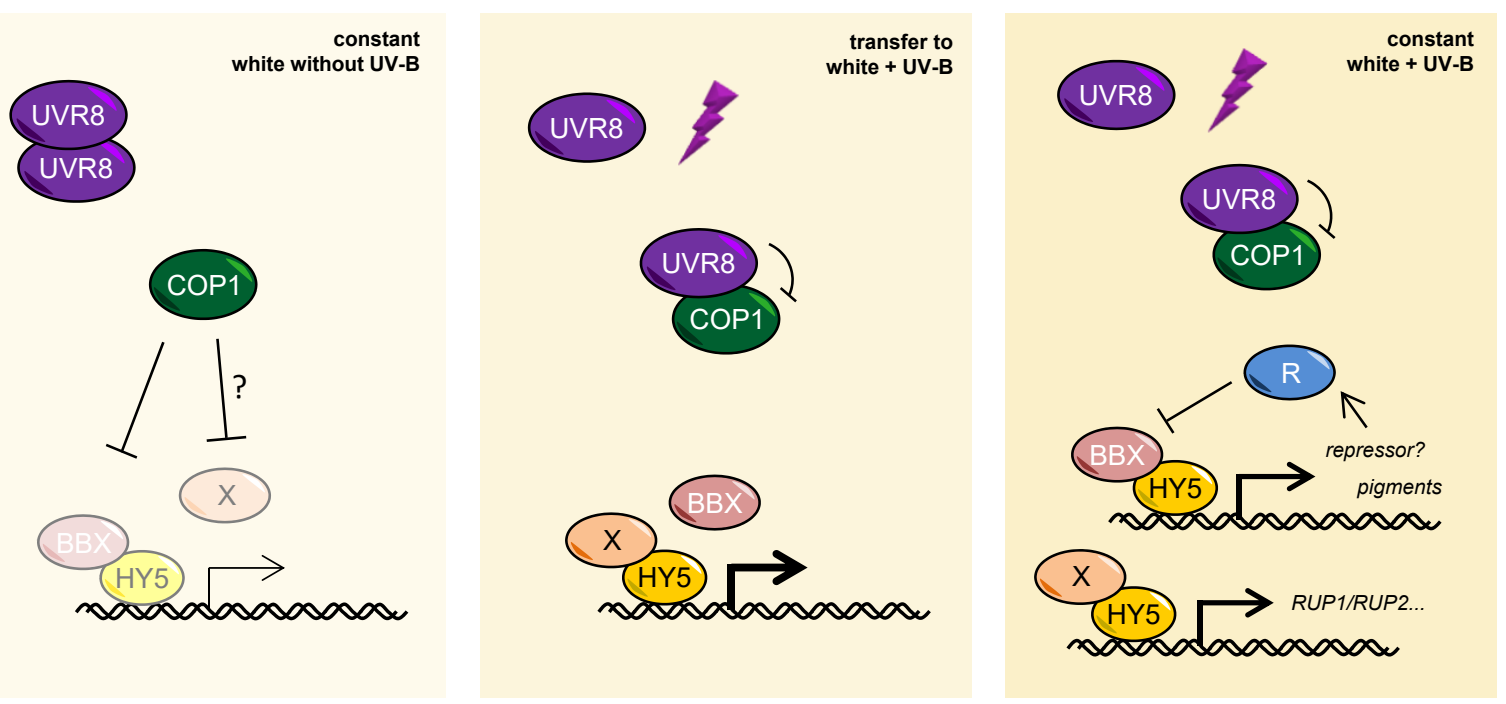

Figure 8 BBX proteins are necessary for the expression of some but not all marker genes in white light conditions constantly supplemented with UV-B. A-E, RT-qPCR analysis F3H (A), MYB12 (B), ELIP2 (C), RUP1 (D), and RUP2 (E) expression in wildtype (Col-0), hy5, and bbx20 bbx21 bbx22 seedlings grown for $4 \mathrm{~d}$ in white light or white light supplemented with UV-B. Values of independent measurements, means, and SEM are shown $(n=4)$; shared letters indicate no statistically significant difference between the means $(P>0.05)$. F, Working model for a role of BBX proteins in UV-B photomorphogenesis. Left - Under weak white light conditions devoid of UV-B, UVR8 is inactive and COP1 targets downstream factors, including HY5, BBX proteins, and possibly a hypothetical factor $X$, for degradation. A small residual pool of HY5 and BBX proteins are responsible for the low basal expression of light-induced marker genes and a weak inhibition of hypocotyl elongation. Middle - Upon exposure of seedlings to UV-B, UVR8 represses COP1 activity. This results in the accumulation of COP1 targets: HY5, BBX proteins, and factor X. HY5 increasingly binds promoters of light-responsive genes, and factor $X$ provides it with transcriptional activity, so that the expression of marker genes is strongly induced. BBX proteins play a minor role in this transcriptional induction. Right - In prolonged UV-B conditions, COP1 remains repressed by UVR8, but this is dampened by negative feedback on photoreceptor activity through RUP1/RUP2. HY5 remains highly stable, but BBX protein levels are repressed through the action of a hypothetical repressor (R) that is induced as a part of HY5/BBX-mediated photomorphogenesis. BBX proteins play a role as HY5 coactivators in allowing the sustained expression of genes involved in pigment biosynthesis, whereas other UV-B marker genes are induced by HY5 in a BBX-independent manner. Hypocotyl length inhibition is controlled by both BBX proteins and factor X. 
bioRxiv preprint doi: https://doi.org/10.1101/2021.10.14.464399; this version posted October 14.2021. The copyright holder for this preprint (which was not certified by peer review) is the author/funder, who has granted bioRxiv a license to display the preprint in perpetuity. It is made available under aCC-BY-NC-ND 4.0 International license.

Supplemental Table S1. Primers used in this study

\begin{tabular}{|c|c|c|}
\hline \multicolumn{3}{|l|}{ RT-qPCR primers } \\
\hline$\underline{\text { Name }}$ & Forward (Fw) sequence & $\underline{\text { Reverse (Rv) sequence }}$ \\
\hline CHS & AGCTGATGGACCTGCAGGCATCTTGGC & TGCATGTGACGTTTCCGAATTGTCGAC \\
\hline$R U P 1$ & AAGTGCCTGTTTCCGAGAGA & GTGGATCCCACATTTGAACC \\
\hline RUP2 & TTGTGGATCGGAAAACAACA & CACTGGTCCACACCTGATTG \\
\hline ELIP2 & TATTGACTACACGCAACATCAGAA & GTTTTCTCCCTTTGATAACTCCAT \\
\hline HY5 (Fig. 5) & CCATCAAGCAGCGAGAGGTCATCAA & CGCCGATCCAGATTCTCTACCGGAA \\
\hline HY5 & CAGCAAGCAAGAGAGAGGAAA & CAGCATTAGAACCACCACCA \\
\hline$B B X 20$ & CATCTCCGGTTCTCTCTCACTTCT & CATTCTCTGCATAGTATTGCTCTGTC \\
\hline$B B X 21$ & AACAAGGACAGAACAACAAGAGA & TTAGAAGAAAGAGGAGGAGGAGTG \\
\hline$B B X 22$ & TGGATCATCTAAGGCGGATACT & GACGCTGTAGGTGGAGACTG \\
\hline MYB12 & AACAGGTGGTCACTAATCGCG & TTCGTTGTCTGTTCTCCCTGG \\
\hline$F 3 H$ & CAGGGACGAAGATGAACGGC & AAGCAAAGAAGTCACGAGCG \\
\hline$P P 2 A$ & TATCGGATGACGATTCTTCGTGCAG & GCTTGGTCGACTATCGGAATGAGAG \\
\hline \multicolumn{3}{|c|}{ CRISPR guide RNA duplexes } \\
\hline$\underline{\text { Name }}$ & Forward (Fw) sequence & $\underline{\text { Reverse (Rv) sequence }}$ \\
\hline BBX21 knockout & ATTGTGCGATAAAGAAGAAGCGT & AAACACGCTTCTTCTTTATCGCA \\
\hline BBX21 $\Delta$ Cterm & ATTGACAAGAGATGGTATGATGA & AAACTCATCATACCATCTCTTGT \\
\hline \multicolumn{3}{|c|}{ bbx21-3D genotyping primers } \\
\hline Name & Forward (Fw) sequence & $\underline{\text { Reverse (Rv) sequence }}$ \\
\hline$b b \times 21-3 D \mathrm{GT}$ & $\begin{array}{l}\text { CAGAGTGGTGATGATGATGGAGTGTTAC } \\
\text { C }\end{array}$ & CTCCCCCATTTAGGGAGATAGTAAAATTACC \\
\hline \multicolumn{3}{|c|}{ BBX22 CDS cloning primers } \\
\hline$\underline{\text { Name }}$ & Forward (Fw) sequence & Reverse (Rv) sequence \\
\hline BBX22_attB & $\begin{array}{l}\text { GGGGACAAGTTTGTACAAAAAAGCAGGC } \\
\text { TTCATGAAGATTCAGTGTAACGTTTGTG }\end{array}$ & $\begin{array}{l}\text { GGGGACCACTTTGTACAAGAAAGCTGGGTCT } \\
\text { AGAACCGTCGCCGC }\end{array}$ \\
\hline
\end{tabular}

